\title{
A Fully Coupled Space-Time Multiscale Modeling Framework for Predicting Tumor Growth
}

\author{
Mohammad Mamunur Rahman ${ }^{1}$, Yusheng Feng ${ }^{1, *}$, \\ Thomas E. Yankeelov ${ }^{2,3,4,5}$, and J. Tinsley Oden ${ }^{2,3}$
}

\begin{abstract}
Most biological systems encountered in living organisms involve highly complex heterogeneous multi-component structures that exhibit different physical, chemical, and biological behavior at different spatial and temporal scales. The development of predictive mathematical and computational models of multiscale events in such systems is a major challenge in contemporary computational biomechanics, particularly the development of models of growing tumors in humans. The aim of this study is to develop a general framework for tumor growth prediction by considering major biological events at tissue, cellular, and subcellular scales. The key to developing such multiscale models is how to bridge spatial and temporal scales that range from $10^{-3}$ to $10^{3} \mathrm{~mm}$ in space and from $10^{-6}$ to $10^{7}$ seconds in time. In this paper, a fully coupled space-time multiscale framework for modeling tumor growth is developed. The framework consists of a tissue scale model, a model of cellular activities, and a subcellular transduction signaling pathway model. The tissue, cellular, and subcellular models in this framework are solved using partial differential equations for tissue growth, agent-based model for cellular events, and ordinary differential equations for
\end{abstract}

\footnotetext{
* Corresponding author

Email address: yusheng.feng@utsa.edu (Yusheng Feng)

${ }^{1}$ Center for Simulation, Visualization and Real-Time Prediction, The University of Texas at San Antonio, One UTSA Circle, San Antonio, TX 78249

${ }^{2}$ Center for Computational Oncology,

${ }^{3}$ Institute for Computational Engineering and Sciences,

${ }^{4}$ Departments of Biomedical Engineering and Internal Medicine, The University of Texas at Austin, 201 East 24th St, Austin, TX 78712

${ }^{5}$ Livestrong Cancer Institutes, 2201 E. Sixth St. Austin, TX 78702
}

Preprint submitted to Computer Methods in Applied Mechanics and EngineeringFebruary 21, 2017

(C) 2017. This manuscript version is made available under the Elsevier user license http://www.elsevier.com/open-access/userlicense/1.0/ 
signaling transduction pathway as a network at subcellular scale. The model is calibrated using experimental observations. Moreover, this model is biologicallydriven from a signaling pathway, volumetrically-consistent between cellular and tissue scale in terms of tumor volume evolution in time, and a biophysicallysound tissue model that satisfies all conservation laws. The results show that the model is capable of predicting major characteristics of tumor growth such as the morphological instability, growth patterns of different cell phenotypes, compact regions of the higher cell density at the tumor region, and the reduction of growth rate due to drug delivery. The predicted treatment outcomes show a reduction in proliferation at different rates in response to different drug dosages. Moreover, the results of several 3D applications to tumor growth and the evolution of cellular and subcellular events are presented.

Keywords: cancer modeling, computer simulation, treatment outcome prediction, finite element method, agent-based method, partial and ordinary equations, continuum mixture theory, signaling transduction pathway, network modeling.

\section{Introduction}

One of the major goals in contemporary medical science is to develop patientspecific treatments guided by predictive computational models, which could eventually result in individualized therapies and more predictable outcomes.

5 Mathematical and computational models can conceivably establish relationships among cancer cell proliferation, intrusion, and microenvironment attributes, provided they can be calibrated and validated with in vitro and in vivo experimental data. Multiscale modeling is one of the effective approaches to use these data in investigations of cancerous cell behavior in tissues and in modeling the overall tumor growth phenomenon (Masoudi-Nejad et al., 2015).

There are two major motivations for using multiscale modeling approaches (Feng et al. 2015). One is the hope that the computational models can eventually displace some of the in vivo and in vitro experiments when the biological 
system in question is well characterized. The other is to test hypothesis and make predictions when there is insufficient information about the biological system under study. To build a multiscale model, one can start with the simplest model and incorporate more features (i.e., bottom up approach) (Schnell et al. 2007 ), or develop and select alternative models based on rigorous model selection criteria (Oden et al. 2013). Augmentations can be incorporated into the model to better characterize the biological system. Nonetheless, it is important to avoid making the model too complicated (Oden et al., 2013, Farrell, 2015).

For tumor growth modeling, it is typical to use, at the tissue scale, the balance/conservation laws of continuum physics with models of biological processes known to exist during the growth or decrease of tumors (see, e.g., Roose et al. 25 (2007); Bellomo et al. (2008)). The latter depicts biological functions, such as growth features characterized by destructive and/or proliferating events. When surface energies of interfaces are modeled using phase-field methods, CahnHilliard type fourth-order nonlinear advection-reaction-diffusion equations describing the cell species appear in the model Wise et al. 2008, Hawkins-Daarud 30 et al. 2012, Lima et al., 2015).

It is obvious that a multiscale model should include major biological events at tissue, cellular and subcellular scales in the tumor growth process. In (Lowengrub et al., 2009), the importance of studying tumor morphology is described. Interestingly, the factors that dictate the shape of the tumor also determine its 35 ability to invade, which can serve as predictors of metastasis. Also, interstitial pressure (Tang et al. 2014) and microenvironment (Macklin \& Lowengrub, 2007) are recognized to be important attributes of tumor shape and growth rate. Moreover, the growth process of the tumor can induce morphological instabilities and surface wrinkling (Cao et al., 2012). However, modeling tumor growth by using information at cellular and subcellular scales to determine how tumor growth occurs at the tissue level can be very challenging.

In this paper, a fully coupled space-time multiscale framework for modeling tumor growth is developed. The framework consists of a tissue scale model, a model of cellular activity and growth, and a subcellular signaling pathway 
model. At the tissue scale, a continuum model is constructed in which the biological tissue is represented as a mixture of multiple constituents interacting with each other through mass and momentum exchange. The constitutive equations at the tissue scale account for tissue anisotropy, nonlinear behavior, and thermodynamic consistency. At the cellular scale, events such as cell divi${ }_{50}$ sion, proliferation, and apoptosis are modeled by using agents representing cell clusters with different phenotypes that evolve in accordance with rule-based algorithms. The transition of phenotypes are up-regulated by models of subcellular signaling pathways and microenvironments.

To bridge the spatial scales, a family of models and algorithms is presented that couples, in space and time, tissue scale behavior and the occurrence of various activities at the cellular scale including cell division, cell death, and phenotypical alteration under evolving cellular microenvironment. The cellular scale is further discretized temporally to model the effects of subcellular signaling pathways and their influence on events at the cellular and tissue scales.

We also incorporate the predictions of the outcome of treatments using a targeted chemotherapeutic drug for the mechanistic target of rapamycin (mTOR) signaling pathway in the subcellular scale model which involves a system of ordinary differential equations in various protein and chemical concentrations. The results show major characteristics of tumors, such as the morphological 65 instability, growth patterns of different cell phenotypes, compact regions of the higher cell density at the tumor region, and the reduction of growth rate due to drug delivery. The multiscale models are calibrated and validated using experimental data (Rahman, 2016), which were obtained via magnetic resonance imaging data acquisition Hormuth et al. (2015). The predicted treatment outcomes show a reduction in proliferation at different rates in response to different dosages. The predicted tumor volume progression matches the experimentally observed volume growth.

The remainder of this manuscript is organized as follows: in Sections 2 and 3 , we present the general mathematical framework and its implementation, 75 respectively. In Section 4, we present and discuss the results before concluding 
in Section 5.

\section{Theory and Mathematical Formulation}

\subsection{General Framework}

Biological events at three scales - tissue, cellular and subcellular - are considered. At the tissue scale, PDE-based continuum mixture theory is deployed so that mechanical conservation laws and the second law of thermodynamics are respected. The overall growth rate or morphology are naturally characterized by time-dependent volume integration and 3D sectional contour plots.

At the cellular scale, an agent-based model representing cellular clusters is constructed according to the rules that govern cell division, apoptosis, and other phenotypical transitions. Biologically defined cell phenotypes, cellular states and transition process are included at this scale to model mitosis, quiescence, and hypoxia of a cell in different microenvironments.

At the subcellular scale, both up- and down-regulations by signaling pathways are modeled. In particular, the mTOR pathway - one of the common signaling pathways involved in tumor growth in many type of cancers - is included. The events at the subcellular scale are modeled using a set of ordinary differential equations. Moreover, a family of targeted drugs, known as rapamycin, is introduced in the subcellular model to target mTOR pathway, providing a means to include the drug treatment effects in the model.

To bridge all three scales, the information from the tissue scale is passed to the cellular scale to determine the fate and phenotypes of a cell or cell cluster. Likewise, the behaviors of a cell or cell cluster will, in turn, affect tissue properties. The regulatory effect of subcellular scale events are represented by signaling pathways. The abundance or scarcity of growth factors and inhibitors at the subcellular scale depends on both signaling transduction control information and microenvironments. All bridging scale models in this frameworkfrom tissue to cells and vice versa, from cells to subcellular pathways and vice versa, and from tissue to pathways-- are fully coupled and solved simultaneously 
in both space and time, representing interactions at all scales. Figure 1 shows the general setting of multiscale coupling designed implemented in this paper.

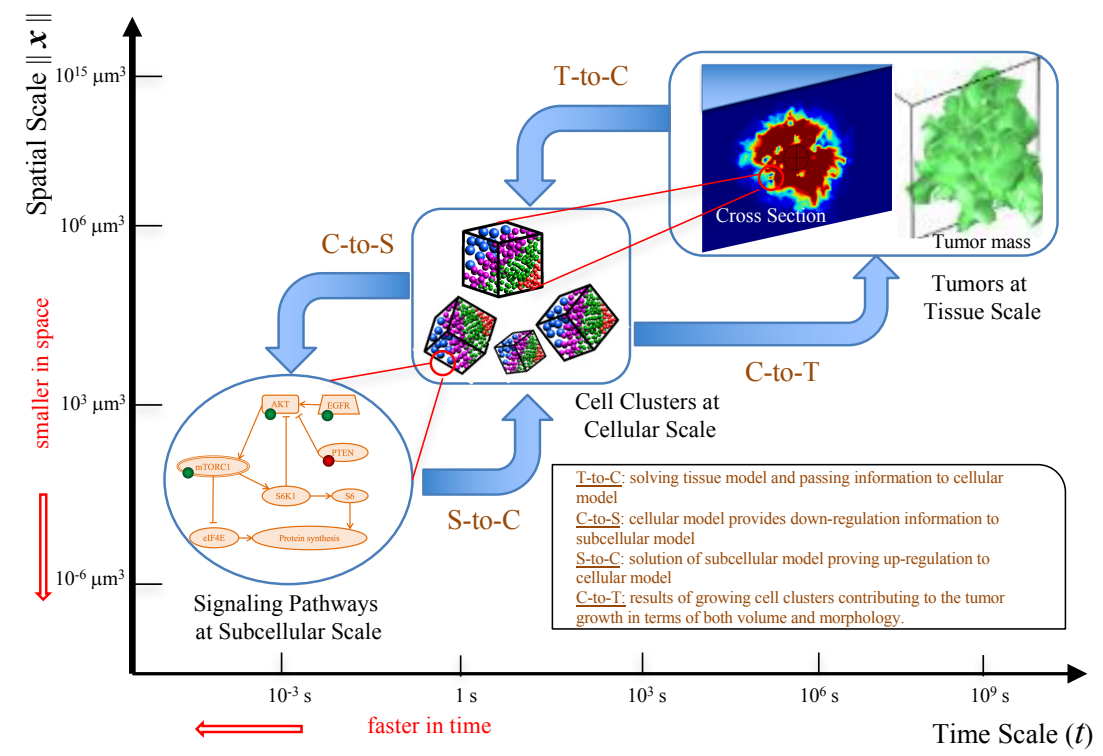

Figure 1: An illustration of the spatiotemporal multiscale modeling framework consisting of the tissue, cellular, and subcellular scales. The domain of spatial scale is a vector space in 3D. It ranges from $10^{-6}$ to $10^{15} \mu \mathrm{m}^{3}$ in volume (micrometers to hundreds centimeters in length ). The time scale ranges from $10^{-3}$ to $10^{9}$ (from microseconds to months and years). The mathematical models at each scale are fully coupled as indicated in the figure, where $T$ denotes as tissue, $C$ as cellular, and $S$ as subcellular.

\section{2. $3 D$ Tissue Scale Model}

A macroscopic tissue is represented by a mixture at each spatial point. Based on mixture theory (Trusdell \& Toupin, 1960, Truesdell \& Noll, 2004), a material body consists of $\kappa$ constituent species that occupy the same region in physical space. At each point of the tissue, we consider that the mixture consisting of $\alpha$ bodies containing constituent $\alpha$, where $\alpha=1, \cdots, \kappa$. 
The volume fraction for each constituent $\alpha$ can be defined as,

$$
n_{\alpha}(\mathbf{X}, t)=\frac{d v_{\alpha}}{d v}
$$

where $\mathbf{X}$ is the position vector, and $t$ represents time, $d v_{\alpha}$ and $d v$ denoting the differential volume element of $\alpha$ constituent and the bulk volume. The saturation condition can be written as

$$
\sum_{\alpha=1}^{\kappa} n_{\alpha}=\sum_{\alpha=1}^{\kappa} \frac{\rho_{\alpha}}{\rho_{\alpha R}}=1
$$

where $\rho_{\alpha}$ denotes the partial mass density of $\alpha$ constituent, and $\rho_{\alpha R}$ denotes the true mass density of the $\alpha$ constituent (see (Bowen, 1976) for detailed kinematics and associated properties).

At the tissue scale, it is assumed that there are four constituents representing (1) tumor cells, (2) healthy cells, (3) extracellular matrix (ECM), and (4) nutrient (oxygen). The overall growth of the tumor, the morphology of the tumor, diffusion of nutrient, and growth-induced solid and fluid stresses are considered in the tissue scale model.

The tumor growth process is governed by mass conservation expressed in the form of the volume fractions of healthy tissues, tumor tissues, and ECM constituents that evolve in time (denoted $n_{H}, n_{T}$, and $n_{F}$, respectively), which, in turn, dictate morphology and density of tumor tissues and their surround125 ings. For growing tissue dynamics, the velocity fields are governed by the momentum equation. Nutrients $(N)$ are assumed to be dissolved in the ECM constituent (interstitial liquid). The convection and diffusion processes of the nutrient constituent is considered separately and represented explicitly in both the momentum and diffusion equations.

The mass balance equations for all three constituents can be expressed as

$$
\frac{\partial \rho_{\alpha}}{\partial t}+\nabla \cdot\left(\rho_{\alpha} \mathbf{v}_{\alpha}\right)=\hat{\rho}_{\alpha}, \quad \alpha=T, H, F
$$

where $\mathbf{v}_{\alpha}$ is the velocity of $\alpha$ constituent and $\hat{\rho}_{\alpha}$ is the mass generation rate of the $\alpha$ constituent per unit volume due to the interaction among all the constituents 
The following convection-diffusion equation governs the nutrient field and its consumption,

$$
d_{a} \frac{\partial c_{N}}{\partial t}=\underbrace{\nabla \cdot\left(c \nabla c_{N}\right)}_{\text {diffusion }}-\underbrace{\mathbf{v}_{F} \cdot \nabla c_{N}}_{\text {convection }}+\underbrace{S_{N}}_{\text {nutrient consumption }}
$$

where $c_{N}$ being the concentration of nutrient (oxygen), $d_{a}$ is the mass coefficient, $c$ is the nutrient diffusion coefficient, $\mathbf{v}_{F}$ is the velocity of the ECM constituent determined by solving the momentum equations (see below) and ${ }_{135} S_{N}=-c_{c} \hat{\rho}_{T}$ represents the consumption of nutrient by the tumor tissue with $c_{c}$ being nutrient consumption rate coefficient.

Next, the balance of momentum is governed by

$$
\nabla \cdot \mathbf{T}_{\alpha}+\rho_{\alpha} \mathbf{b}_{\alpha}+\hat{\mathbf{p}}_{\alpha}=\hat{\rho}_{\alpha} \mathbf{v}_{\alpha}, \quad \alpha=T, H, F
$$

where $\mathbf{T}_{\alpha}$ is the partial Cauchy stress tensor, $\rho_{\alpha} \mathbf{b}_{\alpha}$ is the body force per unit volume, $\hat{\mathbf{p}}_{\alpha}$ is the resultant force exerted on the $\alpha$ constituent by the other constituents per unit volume, which acts as an internal force within the mixture, ${ }_{140} \hat{\rho}_{\alpha}$ represents the rate of change of density of the $\alpha$ constituent, and $\hat{\rho}_{\alpha} \mathbf{v}_{\alpha}$ represents the rate of change of linear momentum due to the mass exchange among the constituents per unit volume. Note that the terms $\hat{\mathbf{p}}_{\alpha}$ and $\hat{\rho}_{\alpha} \mathbf{v}_{\alpha}$ in Eq. (5) are not the same based on the above description. In this equation, all terms are defined as the forces per unit volume. The first term represents the forces directly applied on the $\alpha$ constituent by the other constituents while the second term represents the changing rate of momentum due to the exchange of mass between the $\alpha$ constituent and the other constituents.

The governing Eqs. (3), (4), and (5) are fully coupled. The coupled volume fraction exchange is not only affected by the relative velocity of the movement of the healthy tissue and ECM in the matrix, but also growth factors acting at the interface between the tumor and healthy tissue.

During the growing process, an important factor is the availability of nutrient at the interface. We have considered the concentration of nutrient dissolved in the liquid as the indication of its availability and neglected its volume fraction and force interaction terms. Since mixture as a whole conserves the mass, 
the summation of the mass generation rates for each constituent becomes zero $\left(\hat{\rho}_{T}+\hat{\rho}_{H}+\hat{\rho}_{F}=0\right)$. We have also assumed that the liquid does not participate in the mass exchange. That leads to

$$
\hat{\rho}_{T}=-\hat{\rho}_{H}, \hat{\rho}_{F}=0
$$

The total resultant force exerted on the constituents is zero which can be described as

$$
\hat{\mathbf{p}}_{T}+\hat{\mathbf{p}}_{H}+\hat{\mathbf{p}}_{F}=\mathbf{0} .
$$

Eq. (6) expresses the condition that is generation or loss of mass due to interaction among the constituents. Similarly, Eq. (7) describes the constraint that the net momentum contribution due to interaction among constituents is zero. A transversely isotropic biological tissue featuring a preferred direction is introduced, where the principles of material symmetry impose conditions on the material properties in the domain. The preferred direction can be either constant or time-dependent based on the availability of the nutrient. In three dimensions, this direction can be defined by a unit vector, $\mathbf{A}=\left(A_{x} ; A_{y} ; A_{z}\right)^{T}$, while in two dimensions, it can be represented by an angle $\theta$ as $\mathbf{A}=(\cos \theta ; \sin \theta)^{T}$. The tensor $\mathbf{M}=\mathbf{A} \otimes \mathbf{A}$ is called the structural tensor. The following constitutive relations are defined by the partial Cauchy stress tensors for both solid (tumor and healthy) and fluid (ECM) constituents

$$
\begin{aligned}
\mathbf{T}_{S} & =-n_{S} \lambda \mathbf{I}+\mathbf{T}_{S}^{E}, \\
\mathbf{T}_{F} & =-n_{F} \lambda \mathbf{I}, \\
n_{S} & =n_{T}+n_{H},
\end{aligned}
$$

where the subscript ${ }_{S}$ denotes the solid constituents (i.e., the tumor and healthy constituents), $\lambda$ is the fluid pressure, $\mathbf{I}$ is the identity tensor, and $\mathbf{F}_{S}$ is the deformation gradient for solid constituents. The effective stress of the solid constituents is

$$
\mathbf{T}_{S}^{E}=2 \rho_{S} \mathbf{F}_{S} \frac{\partial \phi_{S}}{\partial \mathbf{C}_{S}} \mathbf{F}_{S}^{T}
$$

where $\mathbf{C}_{S}$ is the right Cauchy-Green tensor defined as

$$
\mathbf{C}_{S}=\mathbf{F}_{S}^{T} \mathbf{F}_{S}
$$


and $\phi_{S}$ is the Helmholtz free energy of ${ }_{S}$ that can be divided into isotropic and transversely isotropic parts as follows Ricken et al. (2007),

$$
\phi_{S}=\left(\frac{n_{S}}{n_{S}^{0}}\right)^{n} \phi_{S, i s o}\left(I_{1}, I_{2}, I_{3}\right)+\phi_{S, t i}\left(J_{4}, J_{5}\right),
$$

where $I_{1,2,3}$ are the principal invariants of $\mathbf{C}_{S}, J_{4,5}$ are the basic invariants of the argument tensors $\mathbf{C}_{S}$ and $\mathbf{M}, \mathbf{M}=\mathbf{A} \otimes \mathbf{A}$ is the structural tensor, $\mathbf{A}=$ $\left[\begin{array}{lll}A_{x} & A_{y} & A_{z}\end{array}\right]^{T}$ is a unit vector pointing to the preferred direction of transversely isotropic solid tumor tissue:

$$
\begin{aligned}
& I_{1}=\operatorname{tr} \mathbf{C}_{S}, \quad I_{2}=\operatorname{tr}\left(\operatorname{det}\left(\mathbf{C}_{S}\right) \mathbf{C}_{S}^{-T}\right), \quad I_{3}=\operatorname{det} \mathbf{C}_{S}=J_{S}^{2}, \\
& J_{4}=\operatorname{tr}\left(\mathbf{C}_{S} \mathbf{M}\right), \quad J_{5}=\operatorname{tr}\left(\mathbf{C}_{S}^{2} \mathbf{M}\right) .
\end{aligned}
$$

The isotropic part of the Helmholtz free energy is assumed to be of the form Ogden (1997),

$$
\phi_{S, i s o}=\frac{1}{\rho_{S}^{0}}\left[\lambda_{S} \frac{1}{2}\left(\log J_{S}\right)^{2}-\mu_{S} \log J_{S}+\frac{1}{2} \mu_{S}\left(I_{1}-3\right)\right],
$$

where $\lambda_{S}$ and $\mu_{S}$ are Lamé's constants for solid constituents. The transversely isotropic part is assumed as Balzani et al. (2006); Ricken et al. (2007),

$$
\phi_{S, t i}= \begin{cases}\frac{1}{2 \rho_{S}^{0}} \alpha_{1}\left(J_{4}-1\right)^{\alpha_{2}} & \text { for } J_{4} \geq 1 \\ 0 & \text { otherwise }\end{cases}
$$

where $\alpha_{1} \geq 1$ and $\alpha_{2}>1$ are parameters related to stiffness of the preferred direction A. Based on standard arguments in continuum mechanics, the Cauchy stress in the solid constituents is derived as,

$$
\mathbf{T}_{S}^{E}=2 \rho_{S} \mathbf{F}_{S} \frac{\partial \phi_{S}}{\partial \mathbf{C}_{S}} \mathbf{F}_{S}^{T}=J_{S} \frac{n_{S}}{n_{S}^{0}}\left[\left(\frac{n_{S}}{n_{S}^{0}}\right)^{3} \mathbf{T}_{S, i s o}^{E}+\mathbf{T}_{S, t i}^{E}\right]
$$

where

$$
\begin{aligned}
\mathbf{T}_{S, i s o}^{E} & =\frac{1}{J_{S}}\left[2 \mu_{S} \mathbf{K}_{S}+\lambda_{S}\left(\log J_{S}\right) \mathbf{I}\right] \\
\mathbf{T}_{S, t i}^{E} & =\frac{1}{J_{S}} \alpha_{1} \alpha_{2}\left[\operatorname{tr}\left(\mathbf{C}_{S} \mathbf{M}\right)-1\right]^{\alpha_{2}-1} \mathbf{F}_{S} \mathbf{M F}_{T}^{S}
\end{aligned}
$$


Here $\mathbf{K}_{S}=\frac{1}{2}\left(\mathbf{F}_{S} \mathbf{F}_{S}^{T}-1\right)$ is the Karni-Reiner strain tensor (Ehlers \& Bluhm, 2013). The interaction force $\hat{\mathbf{p}}_{F}$, i.e., the force applied by the combined solid (tumor and healthy) constituents on the ECM constituent, is given as

$$
\begin{gathered}
\hat{\mathbf{p}}_{F}=\lambda \nabla n_{F}-\mathbf{S}_{F} \mathbf{w}_{F S}, \\
\mathbf{S}_{F}=\frac{\mu_{F R}}{k_{S}^{0}}\left(\frac{n_{F}}{n_{F}^{0}}\right)^{m}[(1-\beta) \mathbf{I}+\beta \mathbf{M}]^{-1},
\end{gathered}
$$

where $\mathbf{w}_{F S}:=\mathbf{v}_{F}-\mathbf{v}_{S}$ is the seepage velocity. $\mathbf{S}_{F}$ describes the permeability tensor of the ECM constituent in connection to the relative velocity, $m$ denotes a dimensionless material parameter, $\mu_{F R}$ the shear viscosity of the fluid, $k_{S}^{0}=$ $k_{S}^{0}\left(n_{H}\right)$ the intrinsic permeability, $\beta$ is the parameter quantifying the relative importance of transversely isotropic symmetry compared to isotropy.

The rate of mass exchange between tumor and healthy constituents can be defined as

$$
\hat{\rho}_{T}(\mathbf{X}, t)=\epsilon(\mathbf{X}, t) \gamma_{\text {prolif }}(\mathbf{X}, t) c_{N}(\mathbf{X}, t) f(t),
$$

where $\epsilon(\mathbf{X}, t)$ is the interface function between tumor and healthy constituents defined by Eq. (19). The $\gamma_{\text {prolif }}(\mathbf{X}, t)$ is an important bridging function among tissue, cellular and subcellular scales. It depends on the outcome of the mitosis activities from the cellular scale model (see Eq. (24)), which in turn depends on the availability of proteins promoting growth from the subcellular scale model. The $c_{N}(\mathbf{X}, t)$ is the concentration of nutrient defined by Eq. (4) and $f(t)$ is a time dependent growth function defined by Eq. 20).

The rate of mass exchange between tumor and healthy regions is proportional to the gradient of volume fraction for tumor constituent. Thus, the interface function $\epsilon$ can be defined as

$$
\epsilon(\mathbf{X}, t)=\left(\nabla n_{T}\right) \cdot\left(\nabla n_{T}\right) .
$$

We define the growth function as

$$
f(t)=\gamma_{0}+\gamma_{1}\left(\frac{t}{\tau_{d}}\right)
$$


where $\gamma_{0}$ and $\gamma_{1}$ are two parameters to be calibrated using experimental data the characteristic time. In this model, $\tau_{d}=1$ day if the growth of the tumor is measured in days.

To summarize, there are seven unknowns $n_{T}, n_{H}, \lambda, c_{N}, v_{S X}, v_{S Y}, v_{S Z}$ representing the volume fraction of tumor and healthy tissues, interstitial pressure, nutrient concentration, and the velocity field components in the $X, Y, Z$ directions.

\subsection{Agent-Based Cellular Scale Model}

We assume that, with appropriate choices of parameters, the tissue scale model presented in the previous section provides a valid characterization of the volume fractions of tumor and healthy cells and of the nutrient concentration $c_{N}$ at every point $\left(\mathbf{X}, t_{\text {tissue }}\right)$ in the space-time domain. The symbol $\mathbf{X}$ denotes the point in the tissue domain $\Omega_{\text {tissue }}$ and $t_{\text {tissue }}$ a time variable measured in the tissue time scale $\left(t_{\text {tissue }} \in\left[0, \tau_{\text {tissue }}\right)\right)$. For each $\left(\mathbf{X}, t_{\text {tissue }}\right) \in \bar{\Omega}_{\text {tissue }} \times$ $\left[0, \tau_{\text {tissue }}\right)$, we consider a volume element $\Omega_{\text {cell }}\left(\left(\mathbf{X}, t_{\text {tissue }}\right)\right)$ which contains a collection of cells refer to as a cell cluster, in which events occur in a much smaller time scale $t_{\text {cell }} \in\left[0, \tau_{\text {cell }}\right)$. In turn, events within $\Omega_{\text {cell }}\left(\left(\mathbf{X}, t_{\text {tissue }}\right)\right)$ are orchestrated by subcellular phenomena which evolve on a still smaller time scale $t_{\text {subcell }} \in\left[0, \tau_{\text {subcell }}\right)$.

The biological events evolving in each volume element are modeled using an agent-based (or rule-based) paradigm which has the following attributes:

(i) There are two types of cells in each cluster: healthy cells and tumor cells. The tumor cells can exist in one of three states (or phenotypes): proliferative, quiescent (predominantly hypoxic), and nonviable (predominantly necrotic). The number of healthy cells in $\Omega_{\text {cell }}\left(\left(x, t_{\text {tissue }}\right)\right)$ is denoted by $C_{H}\left(\mathbf{X}, t_{t i s s u e}, t_{c e l l}\right)$ and can vary over a cell-time span $t_{c e l l}$.

We shall write $C_{H}$ for this function. Likewise, the number of tumor cells is denoted $C_{T}\left(=C_{T}\left(\mathbf{X}, t_{\text {tissue }}, t_{c e l l}\right)\right)$, where $C_{T}=C_{P}+C_{Q}+C_{N V}$ being the 
number of proliferative, quiescent, and nonviable cells, respectively. The total number of cells per volume element is $C_{\text {total }}=C_{H}+C_{P}+C_{Q}+C_{N V}$. The collection of different types of cells- healthy, proliferative, quiescent, and nonviable- form a cell cluster that reside in a volume element $\Omega_{\text {cell }}\left(\left(\mathbf{X}, t_{\text {tissue }}\right)\right)$.

(ii) All human healthy cells may undergo mitosis (cell division) or apoptosis (cell death), but in a cluster containing only healthy cells, there can be no growth; i.e., then $C_{\text {mito }}=C_{\text {apop }}, C_{\text {mito }}$ being the number of cells undergoing mitosis and $C_{\text {apop }}$ being the number of cells undergoing apoptosis. This is due to the fact that we assume no net growth in a matured organ.

(iii) When the microenvironment in which a cell cluster resides (the nutrient level, neighboring cell types, available growing space, etc.) is unfavorable to tumor proliferation, proliferative cells become quiescent (denoted symbolically as $C_{P} \rightarrow C_{Q}$ ), and when the microenvironment is very harsh (i.e., the nutrient concentration is nearly to be zero) the quiescent cells become nonviable $\left(C_{Q} \rightarrow C_{N V}\right)$. On the other hand, the quiescent cells can be rejuvenated and become proliferative again if the microenvironment becomes more favorable $\left(C_{Q} \rightarrow C_{P}\right)$.

(iv) The cellular phenotype transformation from $C_{P}$ to $C_{Q}$ (and vice versa; similarly $C_{Q}$ to $C_{N V}$ ) is orchestrated by the microenvironment and by regulatory control mechanisms at the subcellular scale such as transduction signaling pathways, metabolic pathways and genomic regulatory networks. The regulatory pathway network modeling is discussed in the next section, with which the mitotic and proliferative signals are up-regulators to the cellular scale model.

(v) We define two quantities to characterize cellular microenvironment: Cell Favorability Factor, $F_{f v}$, which is related to cell growth, and Cell Survivability Factor, $F_{s v}$, which determines the harshness of the microenvironment, and the availability of nutrients. 
We propose the following characterization of these factors:

$$
F_{f v}(\mathbf{X}, t)=c_{N}(\mathbf{X}, t)\left(C_{P}+C_{Q}\right) C_{\text {total }}^{-1}\left[1-\left(C_{\text {total }} / C_{t, \text { max }}\right)^{2}\right]
$$

where $C_{t o t a l}$ is the total number of cells and $C_{t, \max }$ is the maximum number of tumor cells allowed in the cell volume element $\Omega_{\text {cell }}(\mathbf{X})$, and

$$
\frac{d F_{s v}}{d t}=-\frac{1}{k_{Q}\left(c_{N}(\mathbf{X}, t), t\right)},
$$

where $k_{Q}$ is the number of days quiescent cells survive without nutrient depending not only the nutrient level but also subcellular activities. Thus, $k_{Q}\left(c_{N}(\mathbf{X}, t), t\right)$ is one of the important parameters that bridges the tissue and subcellular scale behaviors. Note that both $F_{f v}$ and $F_{s v}$ are functions of space and time.

The following is a list of rules that determine the transformation of cell phenotypes inside a cell volume element $\Omega_{\text {cell }}$ :

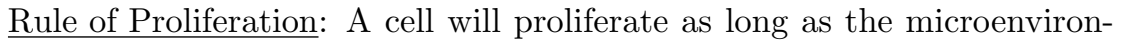
ment is favorable generally meaning that sufficient nutrients and space are available. The number of proliferative cells $\left(C_{P}\right)$ is determined by mitosis, as well as nutrient level is computed from the tissue scale and available space quantified by cell density $\rho_{\text {cell }}$. Initially, $C_{P}$ is set to be equal to $n_{T} / \rho_{\text {cell }}$, where $n_{T}$ is the volume fraction for tumor calculated from tissue scale model.

Rule of Mitosis: Not all proliferative cells will undergo mitosis. For healthy cells, the number of mitotic cells is equal to the number of apoptotic cells. Thus, the net increase is zero. However, for tumor cells, the number will increase and differentiate into different phenotypes. In general, tumor cells may divide in an uncontrollable manner and usually may not exhibit contact inhibition (Zhao et al., 2007). On the other hand, the healthy cells are contact inhibitive and stop reproducing in the presence of the tumor cells. As a result, tumor cells start occupying more space in a cell cluster as healthy cells undergo apoptosis and concede space for tumor cells.

To compute the number of tumor cells going through mitosis in a cell volume $\Omega_{\text {cell }}$, the number of mitotic cells are calculated first based on the 
tumor volume fraction $n_{T}(\boldsymbol{x}, t)$ from the tissue scale model, as well as microenvironment and subcellular information:

$$
C_{m i t o}=C_{m i t o, \min }+k_{\text {protein }} C_{m i t o, m o r e}
$$

where

$$
\begin{gathered}
C_{\text {mito }, \text { min }}=\left(\frac{\Delta n_{T}}{n_{T, \text { max }}}\right) C_{t, \text { min }}, \\
C_{\text {mito,more }}=F_{f v}\left(C_{P}-C_{\text {mito }, \text { min }}\right),
\end{gathered}
$$

the increase $\Delta n_{T}(\boldsymbol{x}, t)$ being solely attributed to mitosis at $(\boldsymbol{x}, t), C_{t, \min }$ is the minimum number of tumor cells in $\Omega_{\text {cell }}(\boldsymbol{x}, t)$ over a time interval, $n_{T, \max }$ is the maximum volume fraction at the tissue scale at any point $\boldsymbol{x}$, and $k_{\text {protein }}$ is a parameter for the synthesis of proteins promoting growth in the subcellular scale (see Eq. (38) in next section). The mitotic cells generated in addition to the change in tumor volume fraction $\Delta n_{T}(\boldsymbol{x}, t)$ at the tissue scale are counted by $C_{\text {mito,more }}$ under the favorable (determined from $\left.F_{f v}\right)$ microenvirnment.

The number of mitotic cells and the availability of the growth promoting proteins regulates the growth of the tumor at the tissue scale model via the proliferation parameter $\gamma_{\text {prolif }}$. The relationship is given as follows,

$$
\gamma_{\text {prolif }}(\mathbf{X}, t)=k_{\text {protein }}\left(\frac{C_{\text {mito }}}{C_{\text {total }}}\right) \text {. }
$$

The value of $\gamma_{\text {prolif }}$ is used in Eq. (18) at the tissue scale to determine the tumor growth rate.

Rule of Cell Quiescence: This rule governs the transformation from proliferative cells to quiescent cells $\left(C_{P} \rightarrow C_{Q}\right)$ and vice versa $\left(C_{Q} \rightarrow C_{P}\right)$. The number of cells that are transformed from proliferative cells to quiescent cells are:

$$
C_{P, Q}=k_{P, Q} C_{P}\left(1-\frac{F_{f v}}{F_{f v, t h}}\right), \quad \text { if } F_{f v}<F_{f v, t h},
$$

where $C_{P, Q}$ is the number of proliferative cells transformed into the quiescent cells in the small predefined volume in the microenvironment, and 
$k_{P, Q}$ is a coefficient of transformation between the proliferative and the quiescent cells. The threshold value $F_{f v, t h}$ is defined as the least favorable microenvironment for a cell to be proliferative.

However, the quiescent cells can transform back to the proliferative cells when the microenvironment becomes more favorable (i.e., $F_{f v} \geq F_{f v, t h}$ ). The transformation is determined by,

$$
C_{Q, P}=k_{Q, P} C_{Q}\left(\frac{F_{f v}-F_{f v, t h}}{1-F_{f v, t h}}\right), \quad \text { if } \quad F_{f v} \geq F_{f v, t h},
$$

where $C_{Q, P}$ is the number of quiescent cells transformed into the proliferative cells in the small predefined volume in the microenvironment, and $k_{Q, P}$ is a coefficient of transformation between the quiescent and the proliferative cells.

Rule of Cell Death: For healthy cells, the cell death goes through apoptosis. With consideration of space inhibition, the number of healthy cells go through apoptosis is given by,

$$
C_{\text {apop }}=\left(\frac{C_{h, \max }}{C_{t, \min }}\right) C_{\text {mito,min }}
$$

where $C_{t, \min }$ and $C_{h, \max }$ are the minimum number of tumor cells and the maximum number of healthy cells, respectively, permitted in the cell volume $\Omega_{\text {cell }}$.

For tumor cells, the apoptotic mechanism is lost. Their death is predominantly due to lack of nutrient and other microenvironmental and subcellular factors. One of the subcelluar mechanisms which determines the death of tumor cell is called autophagy, a natural process for self-digestion of the biological cell that plays a critical role in organelle degradation and protein turnover (Laplante \& Sabatini, 2009, Kobayashi, 2014). These mechanisms can be characterized by a series of activities. Each activity can be quantified by its level of activeness. Thus, if $F_{s v} \leq F_{s v, t h}$, the quiescent cells start transforming into nonviable cells:

$$
C_{Q, N V}=k_{Q, N V} C_{Q}\left(1-\frac{F_{s v}}{F_{s v, t h}}\right),
$$


where $C_{Q, N V}$ is the number of quiescent cells transformed into the nonviable cells and $k_{Q, N V}$ is a coefficient of transformation between the quiescent and the nonviable cell, and $F_{s v, t h}$ is a threshold beyond which a cell will starve to death and/or go through autophagy.

Figure 2 illustrates the transitional relationship among proliferative, quiescent, and nonviable cells under various microenvironments. Note that not all proliferative cells are mitotic, as mentioned previously. Biologically speaking, however, most quiescent cells are hypoxic; and most nonviable cells are necrotic.

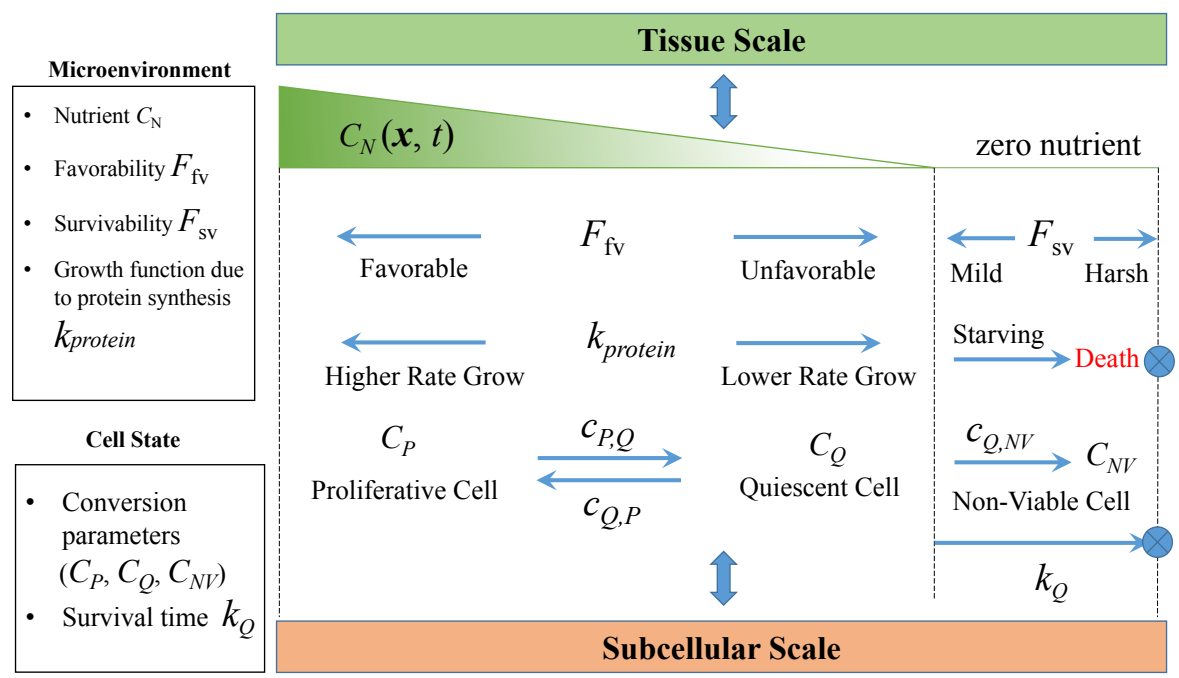

Figure 2: The illustration of relationship among microenvironmental parameters and cell states at the cellular. The microenvironment is quantified by nutrient level $c_{N}$ solved from the tissue scale model, favorability function and survivability function defined in Eqs. 21 and 22 , respectively. The function $k_{\text {protein }}$ is solved at the subcellular scale using Eq. 38. The transition from different cancer cell states (proliferative, quiescent, and non-viable) is controlled by the conversion factors $C_{P, Q}, C_{Q, P}$ and $C_{Q, N V}$ as functions in time. Note that there is no $C_{N V, Q}$ in the picture since non-viable cells are considered as dead cancer cells, and cannot transition back to quiescent cells.

\section{4. subcellular Signaling Pathway Model}

A subcellular scale model frequently involves cell signaling pathways consisting of key biological events such as induction, promotion or inhibition of subcellular signals (Di Ventura et al., 2006). There are many subcellular pathways 
governing cellular activities. Among them, the PI3K/AKT/mTOR pathway is an important regulator for the cell cycle and cell growth (Guertin \& Sabatini, 2007). There are, of course, more than a dozen of proteins, protein complexes, enzymes, and signal receptors (see Fig. 3 (a)) along this pathway. However, we simplify the pathway components by considering the major players that relate to the drug rapamycin as an inhibitor to mTOR complex 1 (mTORC1) (Sharp. 2016).

The simplified pathway (Fig. 3 (b)) components consists of (1) mammalian target of rapamycin complex 1 (mTORC1), (2) protein kinase B (also known as AKT or PKB), (3) epidermal growth factor receptor (EGFR), (4) phosphatase and tensin homolog (PTEN), (5) eukaryotic translation initiation factor 4E (eIF4E), (6) ribosomal protein S6, (7) ribosomal protein S6 kinase beta-1 (also known as S6K1 or p70S6K), and (8) eukaryotic translation initiation factor $4 \mathrm{E}$ (eIF4E). In pathological conditions such as cancer, some components along pathway would either gain (green dot) or loose (red dot) functions, which means the protein level is either over- or under-expressed, respectively.

The following is a set of ODEs formulated using the signaling network model illustrated in Fig. 3 (b):

$$
\begin{aligned}
\frac{d a_{m T O R C 1}}{d t} & =\zeta_{N, m T O R C 1} c_{N}+\zeta_{A K T, m T O R C 1} a_{A K T}-\zeta_{\text {rapa }, m T O R C 1} c_{r a p a} \\
\frac{d a_{A K T}}{d t} & =\zeta_{E G F R, A K T} a_{E G F R}-\zeta_{P T E N, A K T} c_{P T E N}-\zeta_{S 6 K 1, A K T} a_{S 6 K 1} \\
\frac{d a_{S 6 K 1}}{d t} & =\zeta_{m T O R C 1, S 6 K 1} a_{m T O R C 1} \\
\frac{d c_{S 6}}{d t} & =\zeta_{S 6 K 1, S 6} a_{S 6 K 1} \\
\frac{d c_{e I F 4 E}}{d t} & =\zeta_{m T O R C 1, e I F 4 E} a_{m T O R C 1} \\
\frac{d c_{\text {protein }}}{d t} & =\zeta_{e I F 4 E, \text { protein }} c_{e I F 4 E}+\zeta_{S 6, \text { protein }} a_{S 6}
\end{aligned}
$$

Here $c_{N}$ is the concentration of nutrient, $c_{\text {protein }}$ is the concentration of protein that regulates cell proliferation, $c_{S 6}$ is the concentration of activated S6, $c_{e I F 4 E}$ 


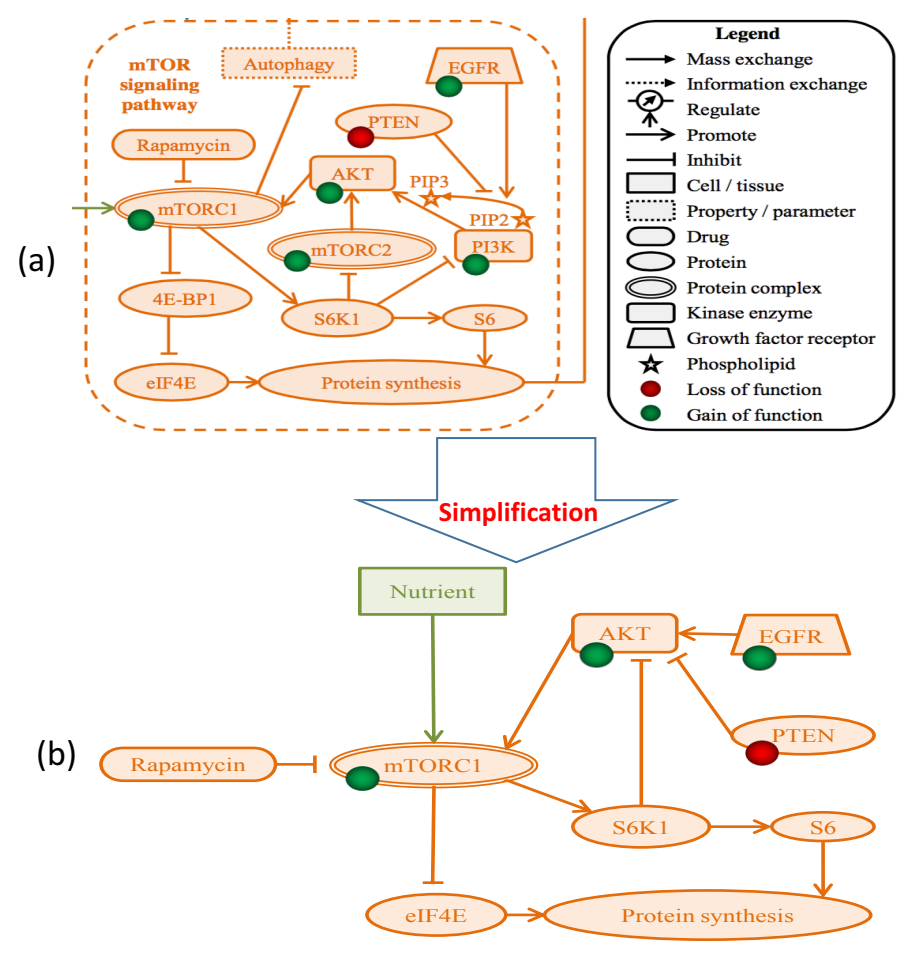

Figure 3: The signaling pathway network model: Panel (a) presents the biological network describing the mTOR pathway (based on Fig. 1 in Polivka \& Janku (2014)), while Panel (b) presents a simplified model based on the assumptions outlined in Section 2.4

is the concentration of activated eIF4E, $c_{\text {rapa }}$ is the concentration of rapamycin, $c_{P T E N}$ is the concentration of activated PTEN, $a_{m T O R C 1}$ is the activity level of mTORC1, $a_{A K T}$ is the activity level of AKT, $a_{S 6 K 1}$ is the activity level of $\mathrm{S} 6 \mathrm{~K} 1, a_{E G F R}$ is the activity level of EGF receptor, and all $\zeta$ with subscripts are the production or degradation constants. Although there are eight components in the simplified pathway, only six ODEs are needed due to uncoupling of EGFR and PTEN. For growth of proteins, S6, eIF4E, rapamycin, and activated PTEN, it is assumed that their concentrations are proportional to their activity level. In the system of ODEs the concentrations are used to quantify the activity 
levels for these quantities. The green and red dots associated with the blocks in Fig. 3 denote gain or loss biological functions of particular proteins or enzymes in cancerous state.

The principal Quantities of Interest in the subcellular model are the activity level of the mTORC1 $\left(a_{m T O R C 1}\right)$ and the concentration of the growth promoting proteins $\left(c_{\text {protein }}\right)$. As discussed earlier, mTORC1 is an inhibitor to autophagy. Thus, we define a time dependent function for autophagy process in terms of $a_{m T O R C 1}$

$$
k_{\text {autophagy }}=\frac{\zeta_{m T O R C 1, \text { autophagy }}}{a_{m T O R C 1}},
$$

where $\zeta_{m T O R C 1, \text { autophagy }}$ is a proportionality constant.

The number of days a quiescent cell survives without nutrient $\left(k_{Q}\right)$ depends on autophagy. More specifically, the cell survival time decreases as autophagy activity increases (i.e., cellular self-digestion process getting faster). Hence, the cell survival time $k_{Q}$ and $k_{\text {autophagy }}$ are inversely related:

$$
k_{Q}=\frac{\zeta_{\text {autophagy }, q}}{k_{\text {autophagy }}}
$$

where $\zeta_{\text {autophagy,q }}$ is a proportionality constant. From Eqs. (35) and (36), we obtain

$$
k_{Q}=\zeta_{m T O R C 1, q} a_{m T O R C 1},
$$

where $\zeta_{m T O R C 1, q}$ is a proportionality constant given by

$$
\zeta_{m T O R C 1, q}=\frac{\zeta_{\text {autophagy }, q}}{\zeta_{m T O R C 1, \text { autophagy }}} .
$$

Therefore, the activity level of the mTORC1 regulates the cell survival rate (recalling Eq. (22)). On the other hand, the growth promoting protein regulates the cell growth. The faster a tumor cell grows, the quicker it reaches to the appropriate size for division. Consequently, the concentration of growth promoting protein regulates mitosis in the cellular scale model. The parameter, $k_{\text {protein }}$ is introduced for the synthesis of the growth promoting protein to regulate mitosis in Eq. 23) as,

$$
k_{\text {protein }}=\zeta_{\text {protein }} c_{\text {protein }}
$$


where $\zeta_{\text {protein }}$ is a proportionality constant. This level of activeness is expressed

using $k_{\text {autophagy }}$ and to be determined by the subcellular scale model in Eqs. 36 . and (37), respectively. It is considered as a regulator of the survival time for the quiescent cells in a harsh environment in which a cell can survive.

\subsection{Physical and Biological Relevance}

At the tissue level, we are interested in the growth phenomena due to evolution and interaction of major biological cell types. Geometrically, the tissue is represented by the union of all elements in the finite element mesh. Within each finite element, it is further divided into very small virtual elements that contain a cluster of cells with different phenotypes. Moreover, volume fractions inside each virtual cell will correspond to each phenotype. To be biologically realistic, we use the same cell size in the virtual cell as that found in the literature. Thus, our multiscale model has the resolution up to cell cluster (collection) level, although not at the single cell level. Moreover, our model is constructed to obey physical conservation laws. The tissue scale model is intended to capture growth processes at the macroscale level. Mixture theory provides a convenient formulation to represent such processes.

Physical parameters are extracted from the literature and used in this model (see Table 1). Only two growth parameters are calibrated at the tissue level, which is justifiable due to the differences of properties from individual to individual. At the cellular scale, our model is constructed to follow the biological processed well-documented in the literature. At the subcellular level, the signaling pathways are based on chemical kinetics models common in stoichiometry of reaction. This results in a network governed by systems of ODEs with nodes representing of concentrations of molecular reactants and products. To ensure the biological relevance, we select the realistic reaction rate constants. The model parameters are listed in Table 1 and used in implementation sections. 


\subsection{A Fully Coupled Spatiotemporal Bridging Method}

\subsubsection{A Multiscale Bridging Algorithm}

All the three spatiotemporal scale models are fully coupled. The local volume fraction or concentration of each constituent solved at the tissue scale is

340 transferred to the cellular scale at every time step. At the same time, the cell proliferation rate, regulated by the cell cycle and more specifically mitosis, is computed and fed back to the tissue scale. The subcellular scale model is also fully coupled with the cellular and tissue scale models. The tissue scale distribution of nutrient is a vital component for the subcellular pathway. On the other hand, subcellular activities, such as the synthesis of protein related to cell proliferation and the autophagy inhibited by mTORC1 protein complex, are transferred to the cellular scale to compute the cell proliferation and cell survivability. The subcellular scale model has smaller time steps than the cellular and tissue scale models. After each cellular scale time step, the solutions are transferred to the subcellular scale and used as the initial conditions of the set of ordinary differential Eqs. 29 to 34 where each cellular scale time step is divided into multiple subcellular scale time steps. The solution is then transferred back to the cellular scale to solve for the next time step.

To design a multiscale algorithm, the key is to transfer modeling information among scales and consider coupling in both space and time. In the framework described in the flow chart (see Fig. 4), a fully coupled algorithm in space is defined. However, the coupling in time must be considered as well. The following integration scheme is developed to account for tissue, cellular and subcellular scales, illustrated in Fig. 1. The events occur at the subcellular scale is much faster than that at either cellular and tissue scale, similar to the events occur at the cellular scale is much faster that that at the tissue scale. Consequently, a numerical integration scheme in time must accommodate such order of magnitude among scales. The general framework for multiscale tumor growth modeling is depicted in Fig. 4 mainly on multiscale coupling in space.

365 To handle multiscale coupling in time, we need to consider the time range that 
events occur at each scale. From the biological viewpoint, typical ranges of time that events occur are different at each scale as described previously. From the computational viewpoint, each time step in the numerical integration at the tissue level needs to be divided into multiple time steps to be compatible at the cellular level. Similarly, each time step at the cellular level need to be further divided into multiple steps at the subcellular level for solving ODE system. The level of division depends on both biological requirement and computational cost. For the sake of saving computational time, the information will be passed across scales until it reaches the time range of the scale above. The implementation on coupling time scale is through numerical integration as discussed in the next section.

\section{Numerical Implementation}

The tissue scale model is governed by a set of partial differential equations (PDEs) defined in Section 2.2. The computational domain includes both the tumor and healthy tissue domains (i.e., $\Omega=\Omega_{T} \cup \Omega_{H} \cup \Gamma_{T H}$, where $\Omega_{T}$ represents the domain of the tumor tissue, $\Omega_{H}$ the domain of the healthy tissue, and $\Gamma_{T H}$ is the interface between the tumor and healthy tissue domains). To solve the PDE system, we need to specify both initial and boundary conditions. Natural boundary conditions are specified at the interface $\Gamma_{T H}$. The governing equations include Eqs. (3), (4), (5) are fully coupled. The value of the maximum volume fraction of the tumor constituent is specified as $n_{T}^{\max }=n_{T}^{0}$ at $\Omega_{T}$. The initial conditions are given as follows,

$$
\left.\left.\begin{array}{l}
\left.\begin{array}{l}
n_{T}=0 \\
n_{H}=n_{H}^{0} \\
\lambda=0
\end{array}\right\} \text { in } \Omega_{H} \\
n_{T}=n_{T}^{0} \\
n_{H}=0 \\
\lambda=0
\end{array}\right\} \text { in } \Omega_{T} \quad \begin{array}{l}
\frac{\partial \mathbf{v}_{\mathbf{T}}}{\partial t}=\mathbf{0} \\
\frac{\partial n_{T}}{\partial t}=\frac{\partial n_{H}}{\partial t}=\frac{\partial p}{\partial t}=0 \\
c_{N}=0 \\
\frac{\partial c_{N}}{\partial t}=0
\end{array}\right\} \text { in } \Omega,
$$




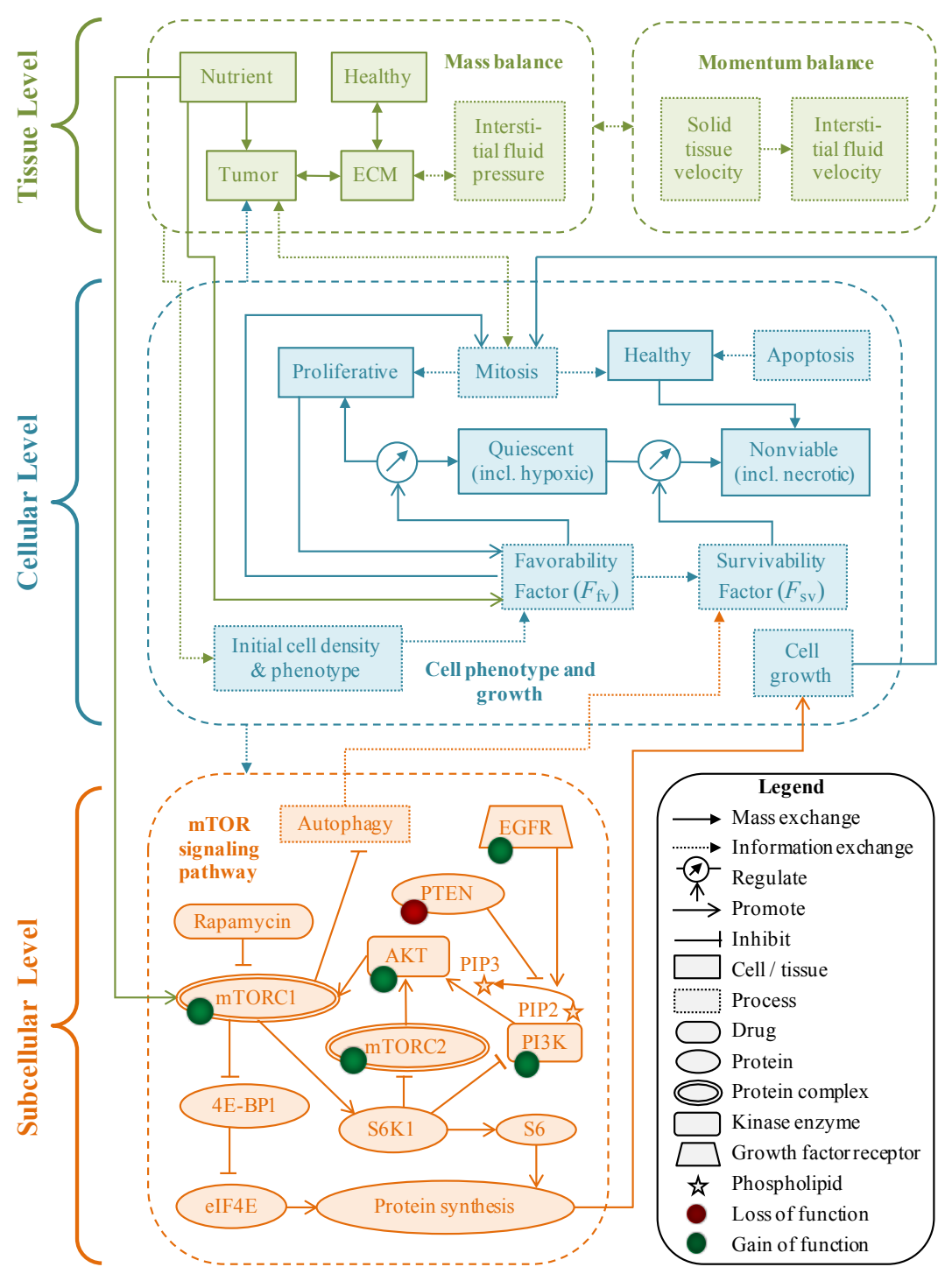

Figure 4: A general framework for a multiscale cancer model: PDE-based tissue scale model, agent-based cellular scale model and ODE-based subcellular model. The fully coupled bridging strategy provides communications between tissue-cellular, cellular-subcellular, tissue-subcellular scales by passing model parameters across the scales. 
and the boundary conditions are prescribed as

$$
\left.\begin{array}{c}
\left.n_{T}=n_{H}\right\} \text { on } \Gamma_{T H} \times(0, T), \\
\nabla n_{T} \cdot \boldsymbol{n}=\nabla n_{H} \cdot \boldsymbol{n}=\nabla p \cdot \boldsymbol{n}=0 \\
\nabla v_{T x} \cdot \boldsymbol{n}=\nabla v_{T y} \cdot \boldsymbol{n}=\nabla v_{T z} \cdot \boldsymbol{n}=0 \\
\nabla c_{N} \cdot \boldsymbol{n}=0 \\
c_{N}=1
\end{array}\right\} \text { on } \Gamma \times(0, T),
$$

where $\Gamma$ is the outer boundary of the large overall $3 \mathrm{D}$ computational domain $\Omega$ and $T$ is the total time period of interest.

Finite element methods are used for solving these governing equations. To begin the solution process, the weak formulations are derived using the standard Galerkin procedure, and implemented in commercial finite element software (COMSOL Multiphysics ${ }^{\circledR}$ 4.4) (Multiphysics, 2013), the governing equations in their weak forms are input into the software. The geometry of computational domain is discretized into an initial mesh, which is further refined near the tumor domain $\Omega_{T}$ so that more accurate solution in the region of interest can be achieved. The size of the initial tumor domain is determined from experimental data.

The model parameters are listed in Table 1. These parameters are physical and biological models and mostly extracted from literature as indicated. The only two parameters $\left(\gamma_{0}\right.$ and $\left.\gamma_{1}\right)$ in the growth model as part of the mixture theory need to be calibrated. The physical meaning of these two parameters is related to the source term $\hat{\rho}_{T}$ (see Eqs. (18) and (20), which can be calibrated using experimental data acquired by magnetic resonance imaging (MRI). The experimental procedure is the same as described in (Hormuth II et al., 2015). The MRI data were acquired over a $32 \times 32 \times 16 \mathrm{~mm}^{3}$ field of view and digitized with a $128 \times 128 \times 16$ matrix, which are used to calculate the tumor volumes over a period of time. Calibration of $\gamma_{0}$ and gamma $_{1}$ are performed both deterministically and stochastically (Rahman, 2016). The uncertainties in 
Table 1: List of model parameter values from literature, along with the values.

\begin{tabular}{|c|c|c|c|c|}
\hline Parameter & Value & Unit & Description & Reference \\
\hline$\mu_{T}$ & $1.34 \times 10^{3}$ & $\mathrm{~Pa}$ & $\begin{array}{c}\text { Shear modulus for tumor } \\
\text { constituent }\end{array}$ & Basser (1992) \\
\hline$\lambda_{T}$ & $6 \times 10^{4}$ & $\mathrm{~Pa}$ & $\begin{array}{c}\text { Lamé's first parameter } \\
\text { for tumor constituent }\end{array}$ & Basser (1992) \\
\hline$k_{T}^{0}$ & $1.43 \times 10^{-1}$ & $\mathrm{~m}^{2}$ & $\begin{array}{c}\text { Intrinsic permeability of } \\
\text { tumor constituent }\end{array}$ & Levin (1980) \\
\hline$\mu_{C R}$ & $1 \times 10^{-3}$ & $\mathrm{Nd} / \mathrm{m}^{2}$ & $\begin{array}{c}\text { Shear viscosity of the } \\
\text { ECM constituents }\end{array}$ & $\begin{array}{c}\text { Ricken et al. } \\
(2007)\end{array}$ \\
\hline$\rho_{T R}$ & 1300 & $\mathrm{~kg} / \mathrm{m}^{3}$ & $\begin{array}{c}\text { True density of tumor } \\
\text { constituent }\end{array}$ & $\begin{array}{c}\text { Nelson et al. } \\
\text { 1971), Bothe }\end{array}$ \\
\hline$\rho_{H R}$ & 1300 & $\mathrm{~kg} / \mathrm{m}^{3}$ & $\begin{array}{c}\text { True density of healthy } \\
\text { constituent }\end{array}$ & $\begin{array}{c}\text { Nelson et al. } \\
\text { (1984), Bothe }\end{array}$ \\
\hline
\end{tabular}


Table 1 Continued

\begin{tabular}{|c|c|c|c|c|}
\hline Parameter & Value & Unit & Description & Reference \\
\hline$\rho_{F R}$ & 993.24 & $\mathrm{~kg} / \mathrm{m}^{3}$ & $\begin{array}{c}\text { True density of ECM } \\
\text { constituent }\end{array}$ & $\frac{\text { Haynes }}{(2013)}$ \\
\hline$n_{T}^{0}$ & 0.20 & - & $\begin{array}{l}\text { Initial volume fraction of } \\
\text { tumor constituent at the } \\
\text { tumor domain } \Omega_{T}\end{array}$ & Bothe et al. \\
\hline$n_{H}^{0}$ & 0.20 & - & $\begin{array}{c}\text { Initial volume fraction of } \\
\text { healthy constituent at } \\
\text { the healthy tissue } \\
\text { domain } \Omega_{H}\end{array}$ & $\begin{array}{c}\text { Bothe et al. } \\
\text { (1984) }\end{array}$ \\
\hline$n_{F}^{0}$ & 0.80 & - & $\begin{array}{l}\text { Initial volume fraction of } \\
\text { ECM constituent at the } \\
\text { whole computational } \\
\text { domain } \Omega\end{array}$ & $\begin{array}{c}\text { Bothe et al. } \\
\text { (1984) }\end{array}$ \\
\hline$c$ & $2 \times 10^{-9}$ & $\mathrm{~m}^{2} / \mathrm{s}$ & $\begin{array}{l}\text { Diffusion coeffficient of } \\
\text { nutrient constituent }\end{array}$ & $\begin{array}{c}\text { Hershey \& } \\
\text { Karhan } \\
(1968)\end{array}$ \\
\hline A & $\left(\frac{1}{\sqrt{3}} \frac{1}{\sqrt{3}} \frac{1}{\sqrt{3}}\right)$ & - & $\begin{array}{l}\text { Preferred direction } \\
\text { vector }\end{array}$ & - \\
\hline$d_{a}$ & $10^{-6}$ & - & Damping coefficient & - \\
\hline$c_{c}$ & $10^{-6}$ & - & $\begin{array}{l}\text { Nutrient consumption } \\
\text { coefficient }\end{array}$ & - \\
\hline$m$ & 2 & - & $\begin{array}{l}\text { Material parameter } \\
\text { related to the } \\
\text { permeability of } \\
\text { combined healthy and } \\
\text { ECM constituents }\end{array}$ & - \\
\hline
\end{tabular}


Table 1 Continued

\begin{tabular}{|c|c|c|c|c|}
\hline Parameter & Value & Unit & Description & Reference \\
\hline$\alpha_{1}$ & 2 & $\mathrm{~Pa}$ & $\begin{array}{l}\text { Stiffness parameter of } \\
\text { the preferred direction }\end{array}$ & - \\
\hline$\alpha_{2}$ & 3 & - & $\begin{array}{l}\text { Stiffness parameter of } \\
\text { the preferred direction }\end{array}$ & - \\
\hline$\beta$ & 0.2 & - & $\begin{array}{l}\text { Relative importance of } \\
\text { transversely isotropic } \\
\text { symmetry compared to } \\
\text { isotropy }\end{array}$ & - \\
\hline$\gamma_{0}$ & $\begin{array}{c}-4 \times \\
10^{-8} \\
5.2 \times 10^{-7}\end{array}$ & $1 / \mathrm{s}$ & Growth factor & calibrated \\
\hline$\gamma_{1}$ & $\begin{array}{c}-2 \times \\
10^{-8} \\
8 \times 10^{-8}\end{array}$ & $1 / \mathrm{s}$ & Growth factor & calibrated \\
\hline
\end{tabular}

The computational domain is determined from the initial diameter of the tumor, the time span of the tumor growth, and the location of the tumor. In this example, the domain is chosen to be a cube with an approximate size of $24 \times 24 \times 24 \mathrm{~mm}^{3}$, which is large enough to avoid any artificial boundary effects. The number of degrees of freedom is 43,197 . Each of these finite elements is spatially discretized further into a set of virtual cell clusters in the cellular scale where the agent-based model is used to determine various cellular activities.

To choose the proper size of each virtual cell inside the finite elements, the actual length scale of biological cells are considered. The number of such division into virtual cells depends on the mesh size. In this implementation, a total number of virtual cells is $912,673(97 \times 97 \times 97)$. The size of the cubic element is determined so that the virtual cell size is equivalent to the typical 
size of cancer cells $\left(\sim 10^{-4} \mathrm{~m}\right)$. Biologically speaking, each cubic element represents small microenvironmental regions and contains a cell cluster, which is a collection of cells of different phenotypes (e.g., healthy, proliferative, quiescent, and nonviable). As the finite element mesh is refined, the size of virtual cell will decrease accordingly. The effect of increasing the virtual cell volume will not affect the cellular activities since the initial virtual cell volume is designed to be equivalent to the biological cell volume. However, the effect of decreasing the virtual cell volume would result in a loss of the biological relevance since the subcellular regulation control would be apply to each phenotype with in the virtual cells (e.g., heathy and cancerous, etc.).

The bridging strategy between the scales in space is illustrated in Fig. 5. Various information such as the geometry of each element, the local volume fraction and concentration of different constituents are passed from the tissue scale to the cellular scale, while the cellular activities such as the phenotypical alteration and cell division are solved at the cellular scale and passed to the tissue scale. The cellular scale model is further discretized temporally into the subcellular scale where an ODE-based technique is used The subcellular scale model is also fully coupled with the cellular and tissue scale models. The time dependent concentrations or activity levels of the subcellular components are solved at this scale using a set of ODEs. To solve the set numerically, an explicit 44 Runge-Kutta $(4,5)$ method (Dormand, 1996), known as the Dormand-Prince method (Dormand \& Prince, 1980), is used.

At the cellular and subcellular scales, a multi-paradigm (Normark, 2013) numerical computing environment $\left(\right.$ MATLAB $\left.^{\circledR}\right)$ is utilized to solve the agentbased algorithm and the set of ODEs, respectively. The coupling between the tissue scale solved using COMSOL Multiphysics ${ }^{\circledR}$ and the cellular and subcellular scales is accomplished by using MATLAB ${ }^{\circledR}$ is communicated via the LiveLink $^{\mathrm{TM}}$ for MATLAB ${ }^{\circledR}$. The spatially discretized computational domain at the cellular scale is used in the subcellular formulation. For each spatial location, the local concentration of various subcellular factors are then computed using time dependent system of ODEs. Each cellular-scale time step is divided 


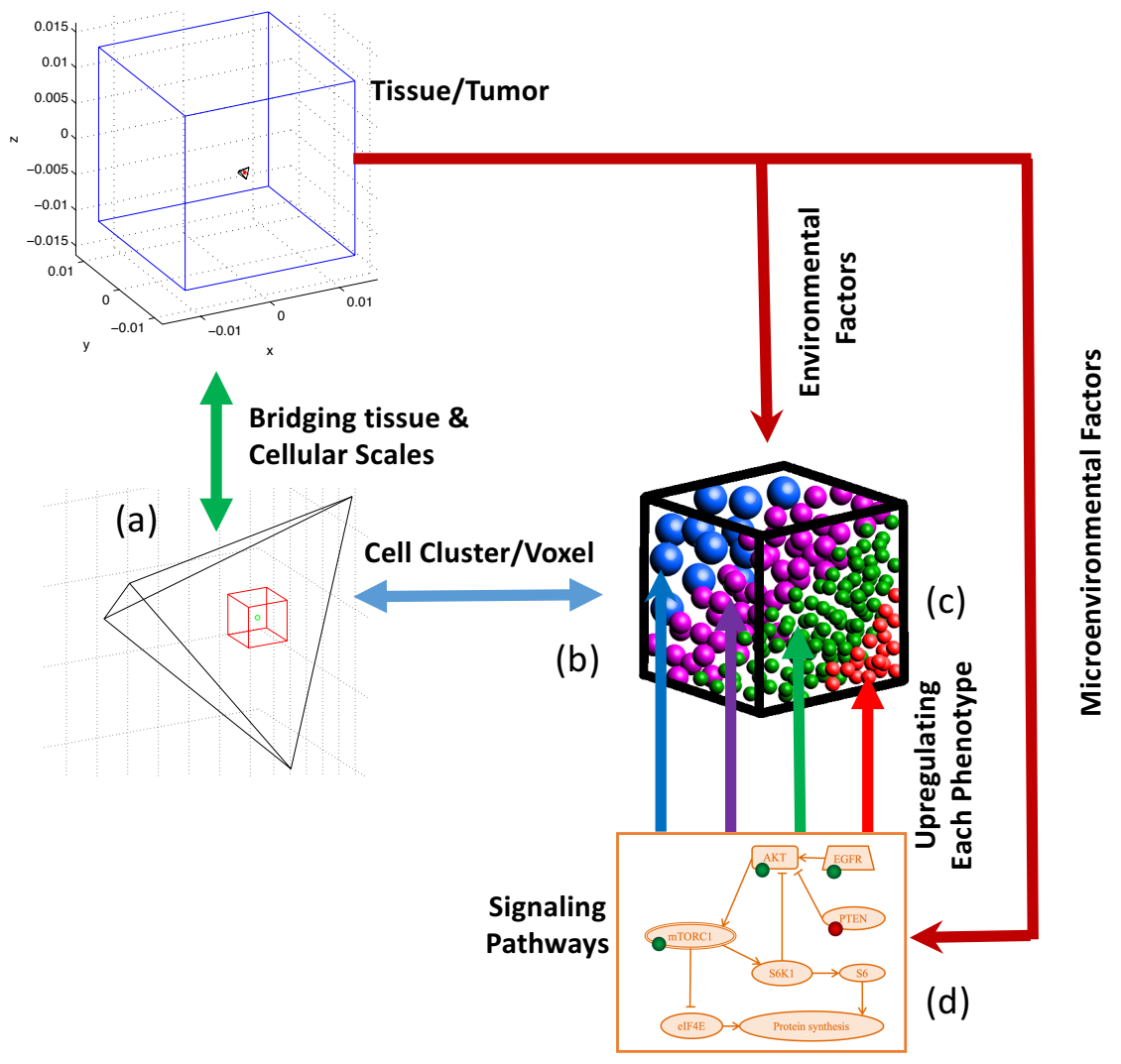

Figure 5: A multi-spatioscale bridging strategy: (a) An illustration of a tetrahedral element (in black) from a tissue scale mesh in the computational domain (in blue). All the other tissue scale elements are hidden in this illustration. (b) A cellular scale volume element $\Omega_{\text {cell }}$ equivalent to MRI voxel size (in red) is illustrated in a zoomed view within the tetrahedral element. All other cellular scale elements are hidden in this illustration. (c) Different cell phenotypes forming a cell cluster are illustrated in a zoomed view of the cellular scale element. Each color represents a phenotype: healthy, proliferative, quiescent, and nonviable cells. (d) the signaling pathway is represented by a biological network with one or multiple pathway(s) up-regulating each cell phenotype 
into smaller steps.

To implement multiscale coupling in time, we established a set of hierarchical relationships across scales. First, select a suitable time intervals $t_{l}, l=$ $0,1,2,3, \cdots$, which will be the time steps for integrating the tissue scale model in the time domain (each time step may not be uniform). Second, the time step at the cellular scale will be determined by $t_{m}=t_{l} / d_{c}, m=0,1,2,3, \cdots$, where $d_{c}$ is a scaling factor to convert a time step at the tissue scale into the time step at the cellular time steps. Third, the time steps at the subcellular scale will be determined by $t_{n}=t_{m} / d_{s}, n=0,1,2,3, \cdots$, where $d_{s}$ is an scaling factor to calculate the time step at the subcellular scale. Fig. 66 illustrates the coupling scheme. Note that, at the tissue level, we only need to solve at time points $t_{l}=t_{0}, t_{1}, t_{3}, t_{5}, \cdots$. At the time points $t_{l}=t_{2}, t_{4}, t_{6}, \cdots$, the up-scale operation is only to collect and formulate the initial conditions to solve tissue scale solutions for the next time step.

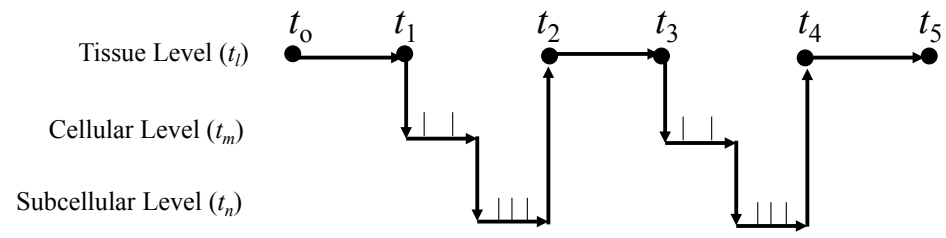

Figure 6: An illustration of time scale coupling algorithm: since the time step at the tissue level is much longer than that at cellular and subcellular level. To bridging the scale, the time step at tissue level must be sub-divided into shorter time steps at the cellular level, and sub-divide further at the subcellular level. However, the hierarchical discretization to bridge scales is only the first step. To design the fully coupled algorithm in time, the most important step is to develop an efficient method to passing information across scales. In the figure, the markers indicate discretized time points while the arrows represent the direction of information flow.

The computer code is verified using the method of manufactured solutions (Roache, 2002). The study shows that the simulation results for different mesh sizes have a good agreement with the exact analytical solution. As the mesh becomes finer, the results become more accurate and indicate that a convergent 
solution can be obtained (see Fig. 7). The relative error is measured between numerical solution and exact solution of a manufactured solution.

The subcellular scale parameters, i.e., the production and degradation constants, are calibrated with the Western blot experiments from the literature using densitometric analysis (Goudar et al. 2005, Wang et al., 2006; Wei et al. 2008 Jiang et al., 2009; Puli et al., 2010; Iwanami et al., 2013, Arcella et al., 2013: Mendiburu-Eliçabe et al., 2014).

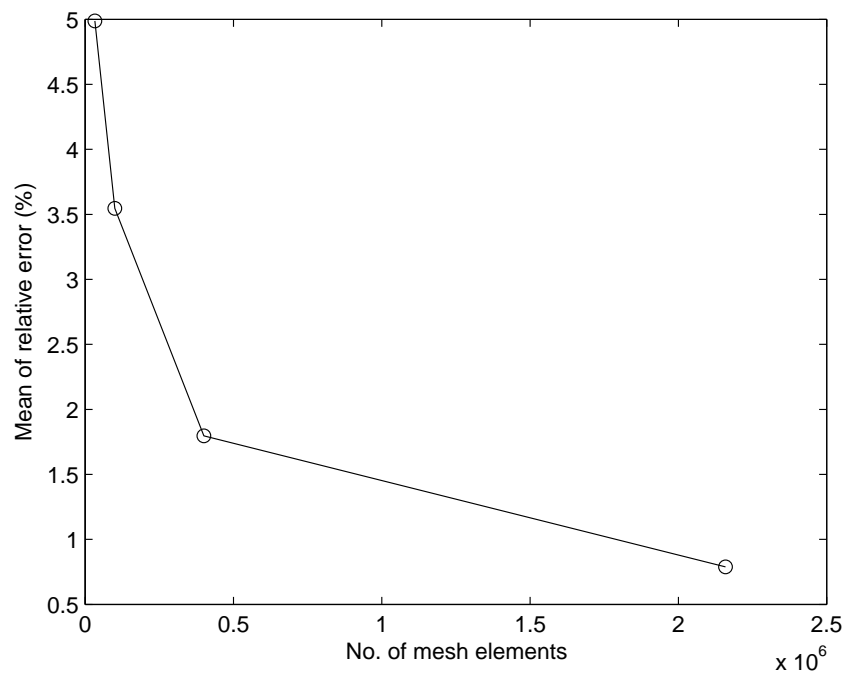

Figure 7: Mean of the relative errors (\%) for different mesh sizes.

\section{Results and Discussion}

\subsection{Results related to Tissue Growth}

The volumetric growth of the tumor in the tissue scale is one the major quantities of interest in this research. The temporal evolution of the tumor from $t=10$ day (when the tumors can be observed by MRI) to $t=18$ day is plotted along with the experimental observation in Fig 8 . To simulate the effectiveness of treatment, one of the commonly used targeted drugs to regulate mTOR pathway is incorporated in subcellular scale model for experiments in 
silico. Various concentrations of rapamycin is tested to discern the effects on the cell proliferation, cell death, and tumor progression. The simulation results show good agreement with experimental data. The effects of the rapamycin is qualitatively consistent with the experimental observations in the literature (Wang et al., 2006, Wei et al., 2008, Puli et al., 2010; Arcella et al., 2013). As the concentration of rapamycin increases, a decline is observed in the overall growth rate of the tumor.

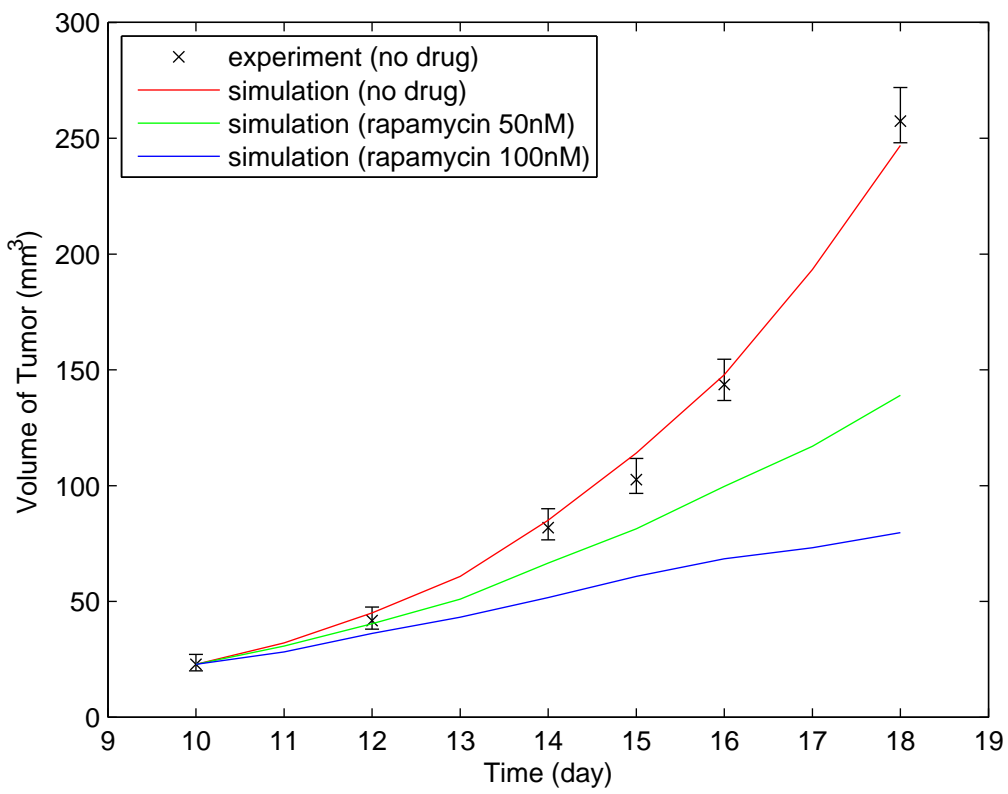

Figure 8: The volumetric growth curves for simulations with different concentrations of rapamycin are compared with the experimental observation with $95 \%$ confidence interval. The green and blue curves are predictions for rapamycin dosage of $50 \mathrm{nM}$ and $100 \mathrm{nM}$, respectively.

For Fig. 9 and 10 , tumor volumes in $3 \mathrm{D}$ and its 2D slice are presented. Each of the figures in Fig. 9 and 10 represents the temporal evolution of the volume at $t=14$ day (on the left side) and $t=18$ day (on the right side) after the simulation starting from $t=10$ day. In each for the figures, there 500 are three different sets of results are shown for different concentrations (dosage) of rapamycin. The top set of two images represent the temporal evolution 
of the volume for no drug delivery. The middle and bottom sets of images represent the temporal evolution of the quantity simulated for $50 \mathrm{nM}$ and 100 $\mathrm{nM}$ of rapamycin dosages, respectively. The numerical results demonstrate the differences in response to different therapeutic dosage levels, as compared to the case that has no treatment.

the difference of response with different chemo-therapeutic dosage level as compared to the case that it has no treatment. demonstrate the differences in response to different chemo-therapeutic dosage levels, as compared to the case that has no treatment. The morphological changes in tumor using 3D plot are evident as shown in Fig. 9. The columns from top down show tumor volume reduction as drug dosage increases at two time points $(\mathrm{t}=14$ and 18 days). The rows from left to right show tumor growth from $t=14$ days to 18 days under the treatment at three dosages level $($ dosage $=0,50 \mathrm{nM}$, and $100 \mathrm{nM})$.

As indicated earlier, Fig. 10 is 2D slices based on 3D contour plot for tumor volume fraction $n_{T}$. The interface between tumor and healthy tissue is observed to be irregular although it started as a smooth sphere at $t=10$ day. The volume of the tumor domain increases over time for all three sets of data. However, the increment in volume for the tumors treated with rapamycin has slower rate than the untreated ones (dosage $=0$ ) comparing with either dosage $50 \mathrm{nM}$ or 100 nM. Tumors with higher drug dosage show slower the growth. To understand the spatial distribution of the volume fraction of tumor constituent $n_{T}$, a cross sectional view along the $Z=0$ axial plane is shown; a gradual declination of $n_{T}$ in terms of area is observed at the interface between tumor and healthy tissues.

\subsection{Results related to Cell Proliferation}

From Fig. 11 to 14 , the cell density (i.e., number of cells per $\mathrm{mm}^{3}$ ) of various types of cells are shown. The proliferating tumor cells push the neighboring healthy cells to occupy the volume.

As shown in Fig. 12, the proliferative cells usually located at the outer most region of the tumor, the interface between the tumor and the healthy cells shown in Fig. 11. Biologically speaking, mTOR complex is a promoter of cell growth 

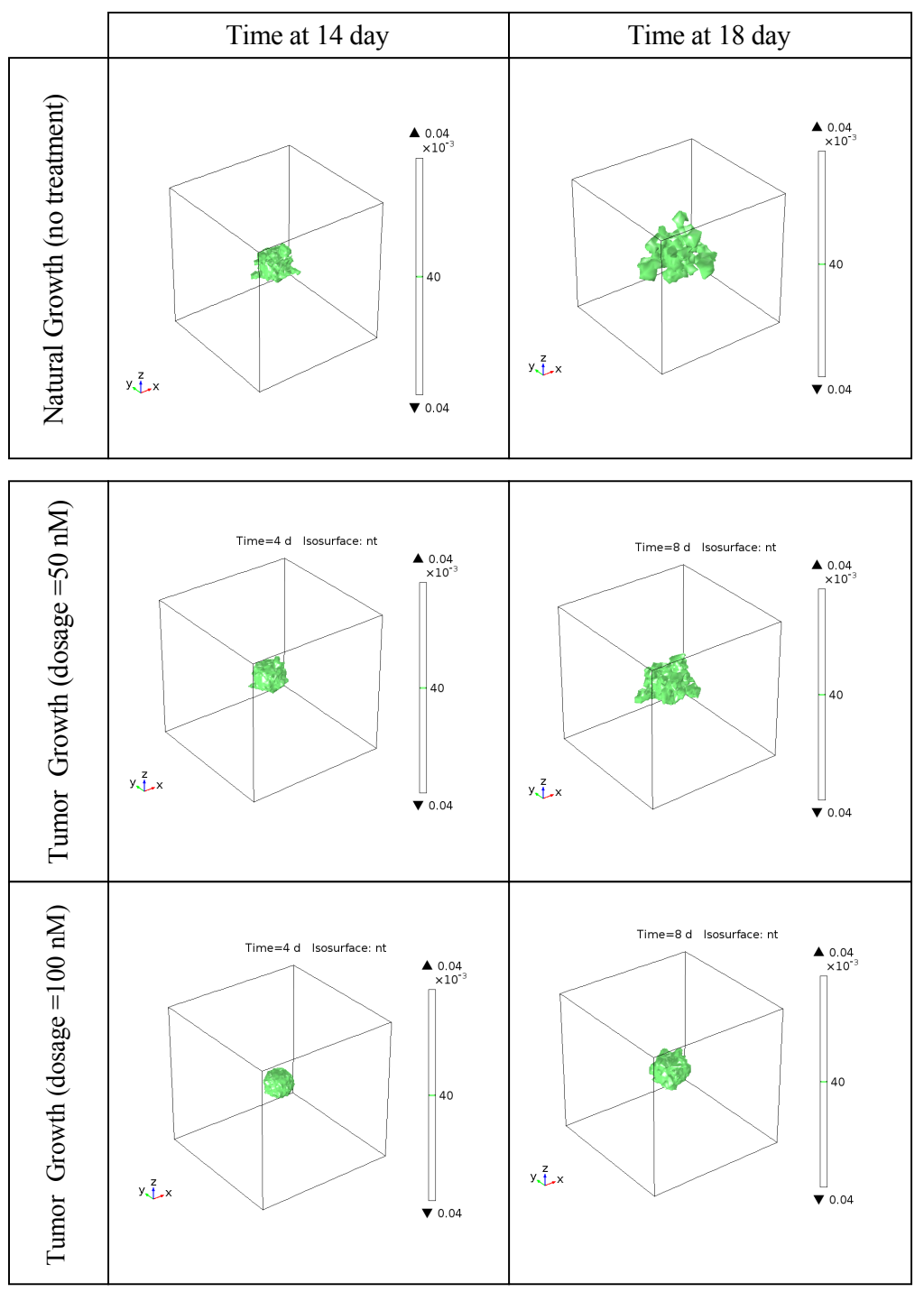

Figure 9: 3D simulation results of total tumor volumes, $\operatorname{vol}(\mathbf{X}, t)$, at two time points over an interval of 10 days: the first row is tumor growth without any drug treatment; the second row is tumor growth with repamycin treatment at a dosage $50 \mathrm{nM}$, and the third row is tumor growth with rapamycin treatment at a dosage $100 \mathrm{nM}$. 

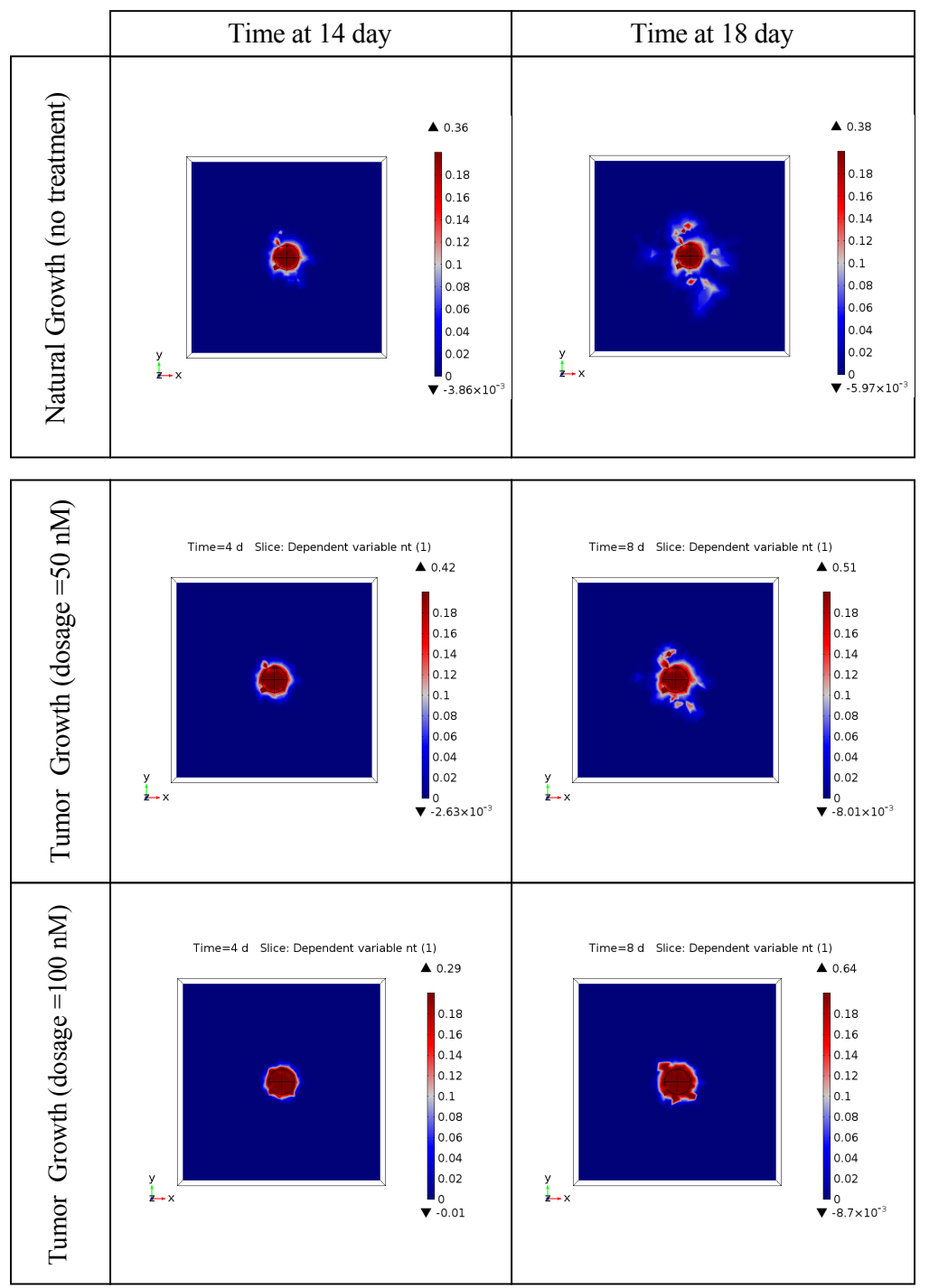

Figure 10: Simulation results of volume fraction distribution of tumor cell constituent $n_{T}(\mathbf{X}, t)$ (cross section $x y$-plane through origin) at two time points over an interval of 10 days, solved at tissue scale: the first row is tumor growth without any drug treatment; the second row is tumor growth with repamycin treatment at a dosage $50 \mathrm{nM}$, and the third row is tumor growth with rapamycin treatment at a dosage $100 \mathrm{nM}$. 
and proliferation. As an inhibitor of mTOR, rapamycin reduces the amount of the proliferative cells, which slows the rate of tumor growth.

Similar to the observation at tissue scale, the scarcity of the nutrient at the inner regions of the tumor transforms the proliferative cells to be hypoxic. Due to nutrient deficiency, these cells cannot grow and become quiescent. Also, insufficient space is another reason why proliferative cells become quiescent shown in Fig 13

When the nutrient is scarce for an extended period of time, the quiescent cells die. The tumor cells can also die due to necrosis, apoptosis, autophagy, and other causes. The cells die due to apoptosis usually locate at the inner core of the tumor where it becomes nutrient deficient. As the rapamycin reduces the tumor size, the necrotic core become smaller. For higher concentrations of rapamycin $\left(c_{\text {rapa }}=100 \mathrm{nM}\right)$, the necrotic core does not form until $t=14$ day.

${ }_{545}$ Although it becomes visible at $t=18$, the size of tumor is much smaller than the other two images for $c_{\text {rapa }}=0 \mathrm{nM}$ and $c_{\text {rapa }}=50 \mathrm{nM}$. The total tumor cell density are shown in Fig 14 , which is the sum of all tumor cell types.

Based on the simulation results, healthy cell distribution has similar patterns to the volume fraction of healthy tissue. The tumor volume at tissue scale can be differentiated into different phenotypes at cellular scale. To illustrate this point, a multiscale results diagram is plotted in Fig 15. Both results of 2D slices at tissue and cellular scale were obtained from 3D simulation. To show effectiveness of targeted drug treatment, a sequence of $2 \mathrm{D}$ slices of total tumor cell density is plotted in terms of drug dosage shown in Fig. 16

\section{Conclusions}

In this study, a general 3D multiple space-time modeling framework for tissue growth is developed. This framework is utilized to model tumor growth for glioblastoma multiforme (GBM). The model accounts for the major events of the growth process of tumors at three spatiotemporal scales: continuum tissue scale coupled with discrete cellular and subcellular scale. The multiscale 

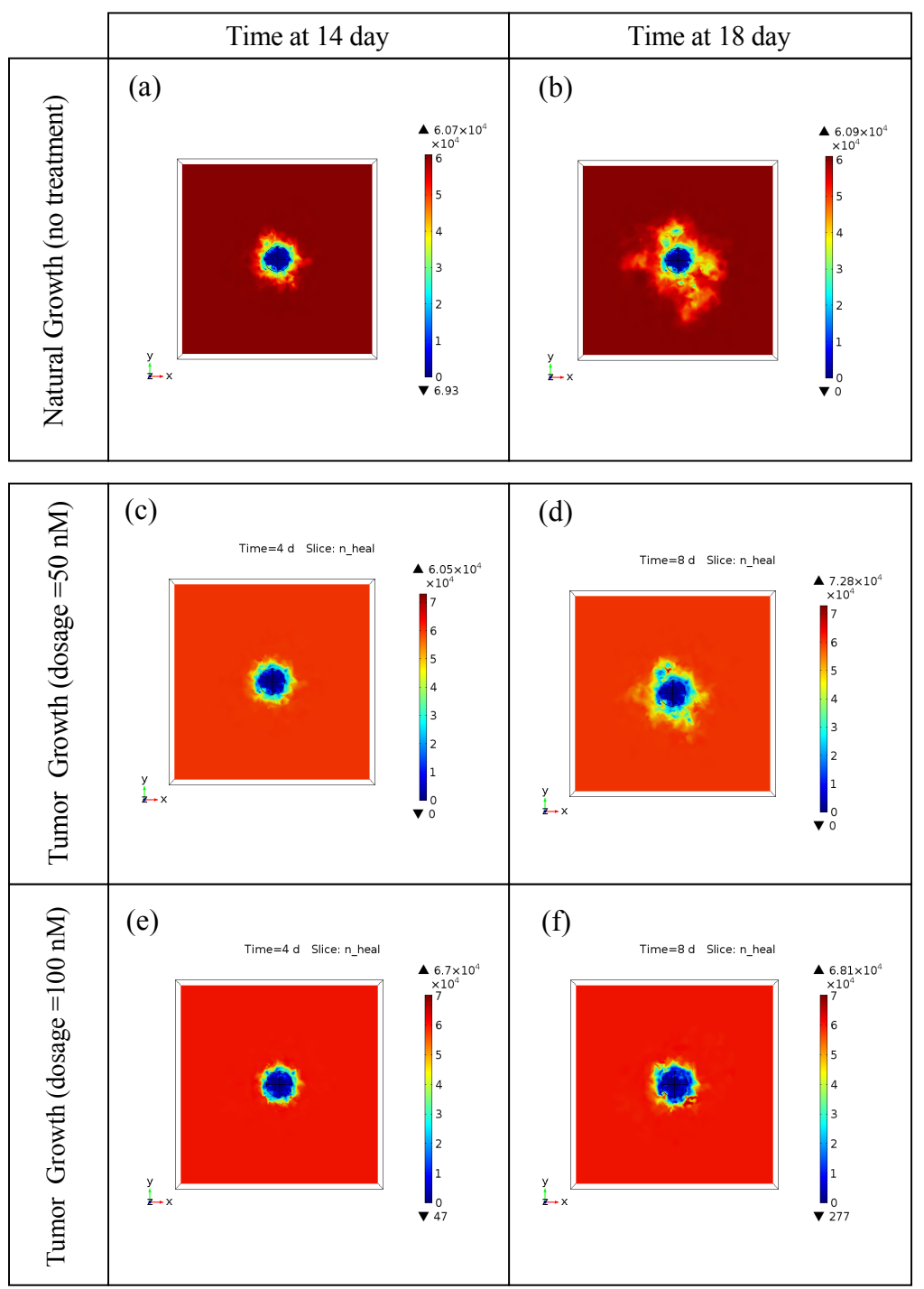

Figure 11: Simulation results of volume fraction distribution of healthy cell type (state) $C_{H}(\mathbf{X}, t)$ (cross section $x y$-plane through origin) at two time points over an interval of 10 days, solved at the cellular scale: the first row is tumor growth without any drug treatment; the second row is tumor growth with repamycin treatment at a dosage $50 \mathrm{nM}$, and the third row is tumor growth with rapamycin treatment at a dosage $100 \mathrm{nM}$. 

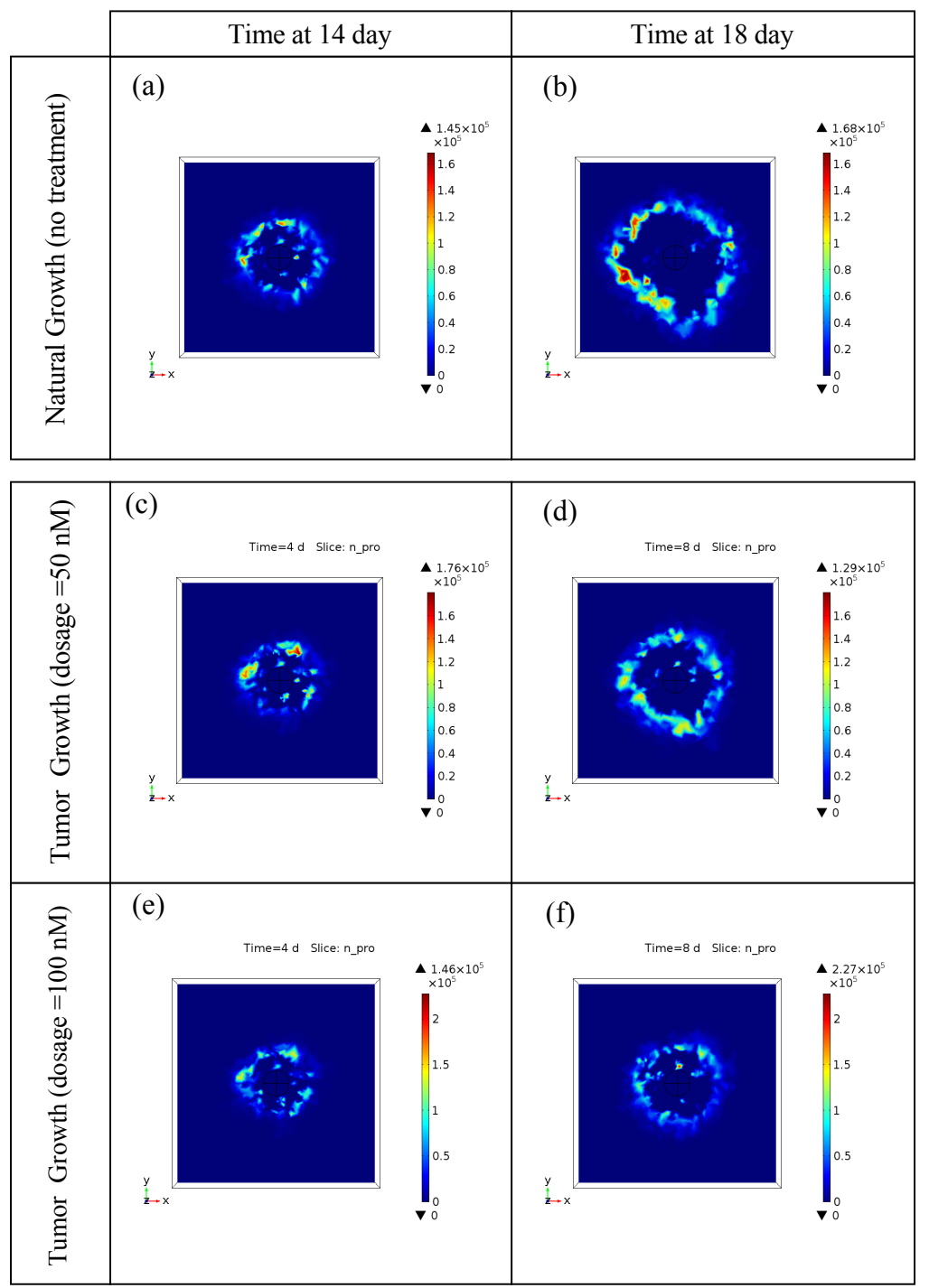

Figure 12: Simulation results of volume fraction distribution of proliferative cell type (state) $C_{P}(\mathbf{X}, t)$ (cross section $x y$-plane through origin) at two time points over an interval of 10 days, solved at the cellular scale: the first row is tumor growth without any drug treatment; the second row is tumor growth with repamycin treatment at a dosage $50 \mathrm{nM}$, and the third row is tumor growth with rapamycin treatment at a dosage $100 \mathrm{nM}$. 

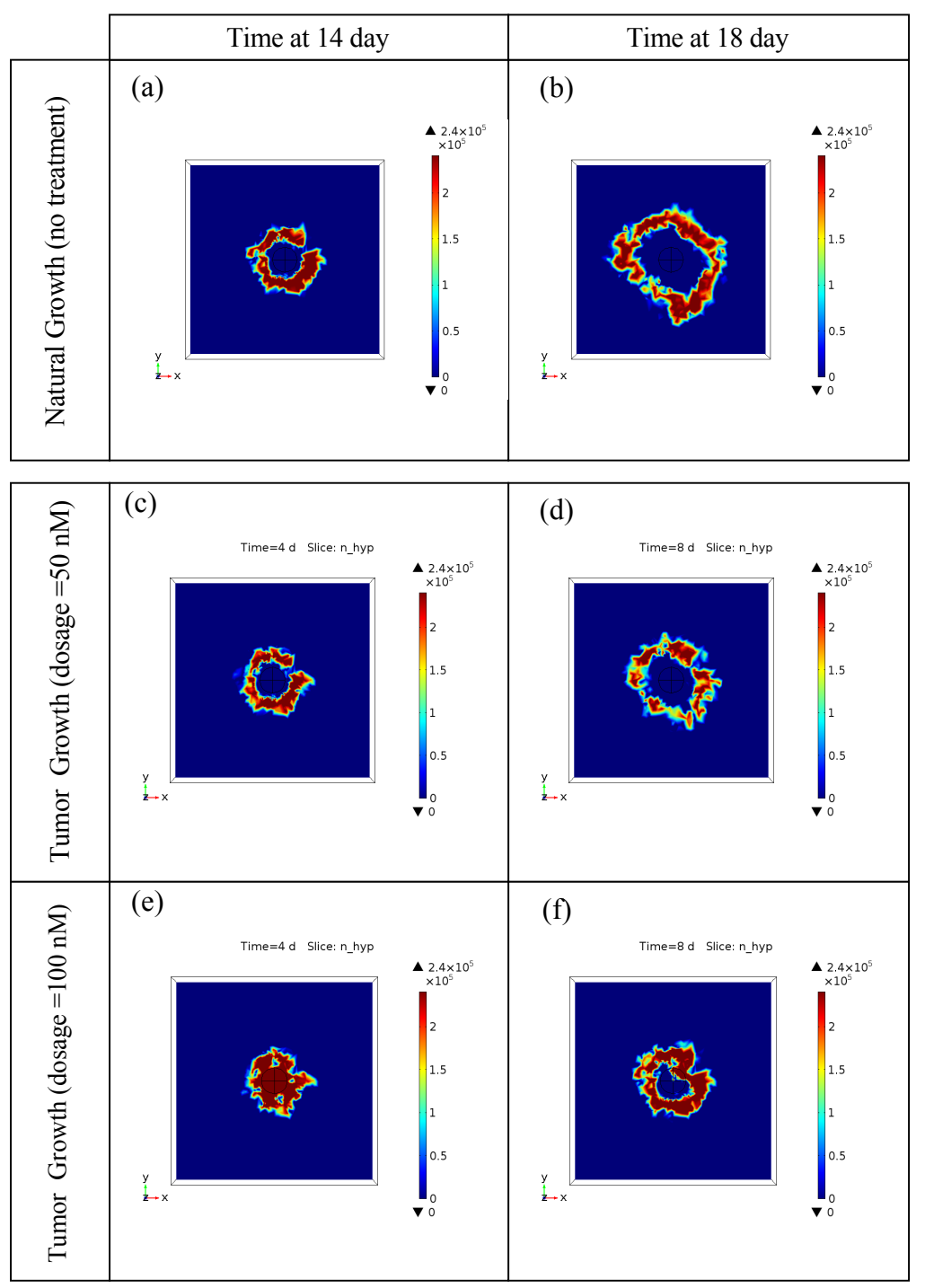

Figure 13: Simulation results of volume fraction distribution of quiescent cell type (state) $C_{Q}(\mathbf{X}, t)$ (cross section $x y$-plane through origin) at two time points over an interval of 10 days, solved at the cellular scale: the first row is tumor growth without any drug treatment; the second row is tumor growth with repamycin treatment at a dosage $50 \mathrm{nM}$, and the third row is tumor growth with rapamycin treatment at a dosage $100 \mathrm{nM}$. 

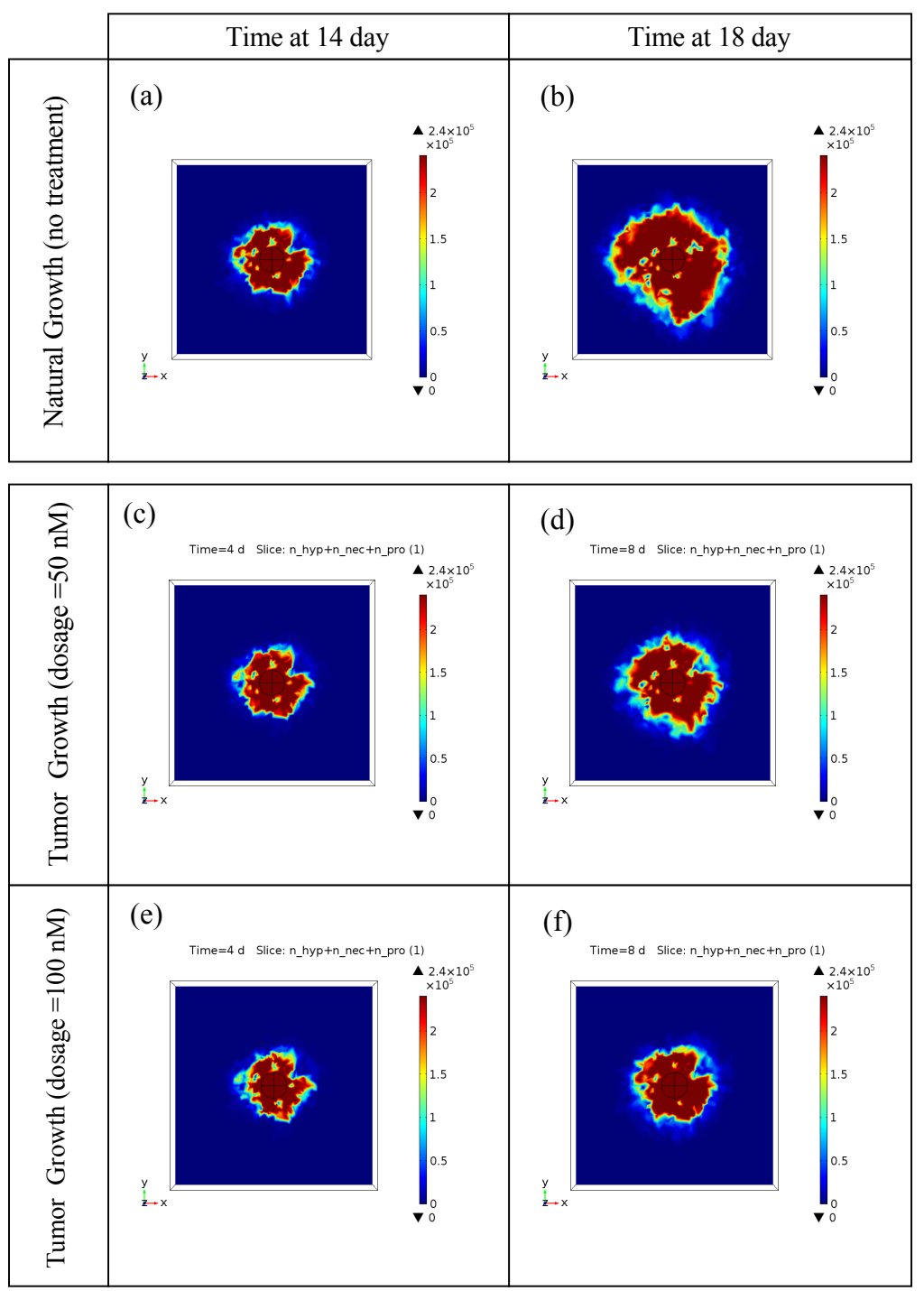

Figure 14: Simulation results of total cell density distribution $C_{\text {total }}(\mathbf{X}, t)$ (cross section $x y$ plane through origin) at two time points over an interval of 10 days, solved at the cellular scale: the first row is tumor growth without any drug treatment; the second row is tumor growth with repamycin treatment at a dosage $50 \mathrm{nM}$, and the third row is tumor growth with rapamycin treatment at a dosage $100 \mathrm{nM}$. 


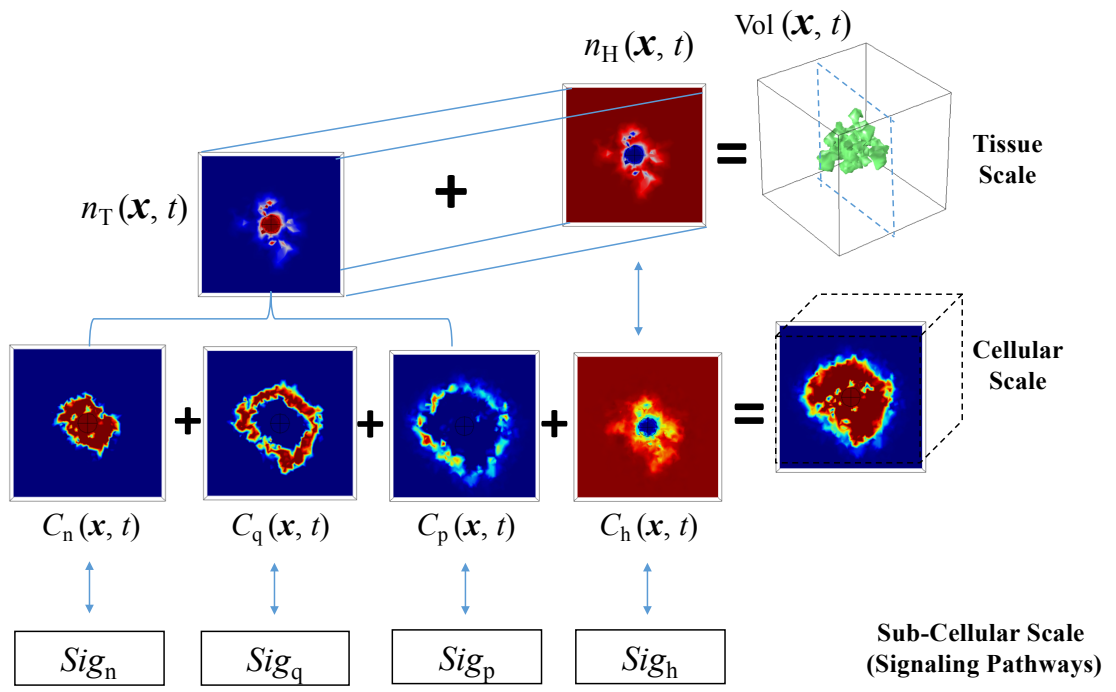

Figure 15: Demonstration of multiscale results. At the tissue scale, the tumor volume is the sum of two constituents (volume fractions for healthy and tumor). At the subcellular scale, the quantities of interested are the cell densities of healthy, proliferative, quiescent, and nonviable cells. The total cell density is the sum of all four cell types (states). At the subcellular scale, there are four corresponding pathways associated with these four cell types.

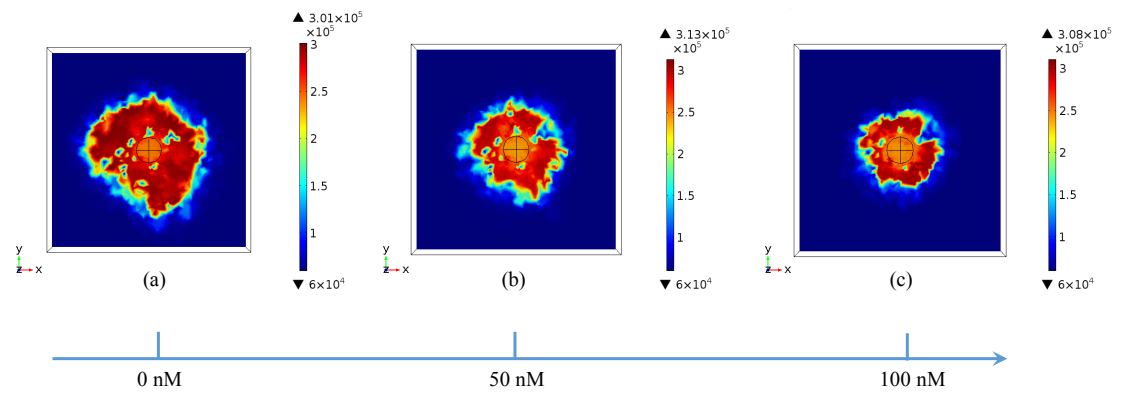

Figure 16: Demonstration of decreasing volume as the chemo-drug dosage increases: (a) without treatment, (b) with rapamycin treatment at $50 \mathrm{nM}$, and (c ) with rapamycin treatment at $100 \mathrm{nM}$. 
framework is designed to incorporate additional constituents at the tissue level, additional cellular phenotypes at the cellular scale and, other signaling pathways at the subcellular scale, through a modular programming implementation.

At the tissue scale, the coupled continuity and momentum equations are used

to account for the effects of both stress and biochemical stimuli in the tumor growth process. The biological tissue is considered as a mixture of multiple constituents where fully nonlinear governing equations with neo-Hookean material behavior are considered to define the constituents of the mixture. The model parameters and material properties are either taken from the literature directly or are calibrated using the experimental data (Hormuth et al., 2015). The numerical simulations are in good agreement with realistic amorphous tumor growth process showing nonuniform growth traits due to neo-Hookean transversely isotropic material properties, the spatial and temporal distribution of nutrient, and the effects of different growth factors.

At the cellular scale, various cellular activities such as the growth, division, phenotypical alteration, and cell death are modeled using a discrete agent-based formulation. In each cell volume element, four phenotypes - healthy, proliferative, quiescent, and nonviable cells - are modeled explicitly. Cells with the same phenotype within the cell volume element are considered to behave the same and treated as one type of agent (i.e., obey the same set rules). The activities of these agents are influenced by the microenvironmental factors depending on the tissue and subcellular scale parameters. The microenvironmental factors are represented by uniformly discretized cubic volumes within each of the finite elements. Each of the microenvironments (cubic volume) hosts a cell cluster. ${ }_{85}$ The tissue scale information, such as the local volume fraction and concentration of the constituents, is transferred to the cellular scale model to simulate the phenotypical alteration, death, and division of cells. These data are in turn fed back to the tissue scale model. subcellular information, such as growth promoting protein synthesis, is transferred from the subcellular scale to the cellular scale.

The effects of the targeted drug rapamycin (eRapa) is investigated using 
the described multiscale framework. In many cancers, including GBM, the rapamycin inhibits the mTOR protein complex and thus regulates tumor cell proliferation and tissue growth processes. Numerical results for different concentrations (dosage level) of rapamycin show the effectiveness of the drug to impede the progression of cancer in terms of tumor volume reduction.

It is important to note that all models at the three scales are physics and biology-based. The resolution of this multiscale model is as accurate up to the cell cluster level. Thus, the mixture theory is a valid representation for the phenomena in this mathematical framework. Similarly, the model at the cellular level is based on the biological laws about how cells behave, by which the rulebased model is constructed. At the subcellular level, the signaling pathways are modeled by ODEs.

In conclusion, the space-time multiscale modeling framework presented in this paper is quite general and can be enhanced by adding more species at the tissue scale, including extra phenotypical cell types at cellular scale and increasing the number of pathways so that one phenotypical cell type can be up-regulated by one or combination of a few signaling pathways, which would be more biologically significant. This model is biologically-driven from signaling pathway, volumetrically-consistent between cellular and tissue scale in terms of tumor volume evolution in time, and biophysically-sound in tissue model by satisfying all conservation laws. The model is capable of predicting the volume of a tumor after calibration with respect to experimental data. It is hoped that this multiscale computational strategy, as presented in this paper, will play an important role for patient-specific prediction of tumor growth and personalized treatment. The future work may include multiple pathways at the subcellular scale or integrate RNA and DNA information to make more patient-specific predictions.

\section{Acknowledgements:}

This research was partially supported by NSF/CREST-HRD \#0932339 (RM 
and YF), the U.S. DOE Office of Science, Office of Scientific Computing Research, Advanced Mathematics program under award DE 50000926 (JTO), NIH/NCI U01CA174706 (TEY), NIH/NCI R01 186193 (TEY), and CPRIT RR160005 (TEY). We thank our colleagues David Sharp, PhD, Ronald Rodriguez, MD, $\mathrm{PhD}$, and Luzhe Sun, $\mathrm{PhD}$, from the University of Texas Health Science Center at San Antonio, who provided insight and expertise that greatly assisted the research in terms of describing cancer cell evolving process of different phenotypes, selecting targeted drug rapamycin for mTOR signaling pathways, and simplifying mTOR pathway for modeling purpose.

\section{References}

Arcella, A., Biagioni, F., Oliva, M. A., Bucci, D., Frati, A., Esposito, V., Cantore, G., Giangaspero, F., \& Fornai, F. (2013). Rapamycin inhibits the growth of glioblastoma. Brain Research, 1495, 37-51.

Balzani, D., Neff, P., Schröder, J., \& Holzapfel, G. A. (2006). A polyconvex framework for soft biological tissues. adjustment to experimental data. International Journal of Solids and Structures, 43, 6052-6070.

Basser, P. J. (1992). Interstitial pressure, volume, and flow during infusion into brain tissue. Microvascular Research, 44, 143-165.

Bellomo, N., Li, N., \& Maini, P. K. (2008). On the foundations of cancer modelling: selected topics, speculations, and perspectives. Mathematical Models and Methods in Applied Sciences, 18, 593-646.

Bothe, H.-W., Bodsch, W., \& Hossmann, K.-A. (1984). Relationship between specific gravity, water content, and serum protein extravasation in various types of vasogenic brain edema. Acta Neuropathologica, 64, 37-42.

Bowen, R. M. (1976). Theory of mixtures. Continuum physics, 3. 
Cao, Y., Jiang, Y., Li, B., \& Feng, X. (2012). Biomechanical modeling of surface wrinkling of soft tissues with growth-dependent mechanical properties. Acta Mechanica Solida Sinica, 25, 483-492.

Di Ventura, B., Lemerle, C., Michalodimitrakis, K., \& Serrano, L. (2006). From in vivo to in silico biology and back. Nature, 443, 527-533.

Dormand, J. R. (1996). Numerical methods for differential equations: a computational approach volume 3. CRC Press.

Dormand, J. R., \& Prince, P. J. (1980). A family of embedded runge-kutta formulae. Journal of Computational and Applied Mathematics, 6, 19-26.

Ehlers, W., \& Bluhm, J. (2013). Porous media: theory, experiments and numerical applications. Springer Science \& Business Media.

Farrell, K. A. (2015). Selection, calibration, and validation of coarse-grained models of atomistic systems. PhD dissertation, The University of Texas at Austin.

Feng, Y., Boukhris, S. J., Ranjan, R., \& Valencia, R. A. (2015). Biological systems: Multiscale modeling based on mixture theory. In Multiscale Modeling in Biomechanics and Mechanobiology (pp. 257-286). Springer.

Goudar, R. K., Shi, Q., Hjelmeland, M. D., Keir, S. T., McLendon, R. E., Wikstrand, C. J., Reese, E. D., Conrad, C. A., Traxler, P., Lane, H. A. et al. (2005). Combination therapy of inhibitors of epidermal growth factor receptor/vascular endothelial growth factor receptor 2 (aee788) and the mammalian target of rapamycin (rad001) offers improved glioblastoma tumor growth inhibition. Molecular cancer therapeutics, 4, 101-112.

Guertin, D. A., \& Sabatini, D. M. (2007). Defining the role of mtor in cancer. 670 Cancer cell, 12, 9-22.

Hawkins-Daarud, A., van der Zee, K. G., \& T. Oden, J. (2012). Numerical simulation of a thermodynamically consistent four-species tumor growth model. 
International Journal for Numerical Methods in Biomedical Engineering, 28, $3-24$.

Hershey, D., \& Karhan, T. (1968). Diffusion coefficients for oxygen transport in whole blood. AIChE Journal, 14, 969-972.

Hormuth, D., Weis, J., Barnes, S., Miga, M., Reicha, E., \& Quaranta, T. (2015). Predicting in vivo glioma growth with the reaction diffusion equation con-

Iwanami, A., Gini, B., Zanca, C., Matsutani, T., Assuncao, A., Nael, A., Dang, J., Yang, H., Zhu, S., Kohyama, J. et al. (2013). Pml mediates glioblastoma resistance to mammalian target of rapamycin (mtor)-targeted therapies. Proceedings of the National Academy of Sciences, 110, 4339-4344.

Jiang, H., Shang, X., Wu, H., Gautam, S. C., Al-Holou, S., Li, C., Kuo, J., Zhang, L., \& Chopp, M. (2009). Resveratrol downregulates pi3k/akt/mtor signaling pathways in human u251 glioma cells. Journal of Experimental Therapeutics \& Oncology, 8, 25.

Kobayashi, S. (2014). Choose delicately and reuse adequately: The newly revealed process of autophagy. Biological $\& 3$ pharmaceutical bulletin, 38, 10981103.

Laplante, M., \& Sabatini, D. M. (2009). mtor signaling at a glance. Journal of cell science, 122, 3589-3594. 
Levin, V. A. (1980). Relationship of octanol/water partition coefficient and molecular weight to rat brain capillary permeability. Journal of Medicinal Chemistry, 23, 682-684.

Lima, E. A., Almeida, R. C., \& Oden, J. T. (2015). Analysis and numerical solution of stochastic phase-field models of tumor growth. Numerical Methods for Partial Differential Equations, 31, 552-574.

Lowengrub, J. S., Frieboes, H. B., Jin, F., Chuang, Y., Li, X., Macklin, P., Wise, S., \& Cristini, V. (2009). Nonlinear modelling of cancer: bridging the gap between cells and tumours. Nonlinearity, 23, R1.

Macklin, P., \& Lowengrub, J. (2007). Nonlinear simulation of the effect of microenvironment on tumor growth. Journal of Theoretical Biology, 245, $677-704$.

Masoudi-Nejad, A., Bidkhori, G., Ashtiani, S. H., Najafi, A., Bozorgmehr, J. H., \& Wang, E. (2015). Cancer systems biology and modeling: Microscopic scale and multiscale approaches. In Seminars in Cancer Biology (pp. 60-69). Elsevier volume 30 .

Mendiburu-Eliçabe, M., Gil-Ranedo, J., \& Izquierdo, M. (2014). Efficacy of rapamycin against glioblastoma cancer stem cells. Clinical and Translational Oncology, 16, 495-502.

Multiphysics, C. (2013). Comsol Multiphysics Reference Manual volume Version 4.4. COMSOL. URL: www. comsol.com.

${ }_{720}$ Nelson, S., Mantz, M., \& Maxwell, J. (1971). Use of specific gravity in the measurement of cerebral edema. J Appl Physiol, 30, 268-271.

Normark, K. (2013). Overview of the four main programming paradigms, .

Oden, J. T., Prudencio, E. E., \& Hawkins-Daarud, A. (2013). Selection and assessment of phenomenological models of tumor growth. Mathematical Models and Methods in Applied Sciences, 23, 1309-1338. 
Ogden, R. W. (1997). Non-linear elastic deformations. Courier Corporation.

Polivka, J., \& Janku, F. (2014). Molecular targets for cancer therapy in the pi3k/akt/mtor pathway. Pharmacology \& Therapeutics, 142, 164-175.

Puli, S., Jain, A., Lai, J. C., \& Bhushan, A. (2010). Effect of combination treatment of rapamycin and isoflavones on mtor pathway in human glioblastoma (u87) cells. Neurochemical Research, 35, 986-993.

Rahman, M. (2016). Multiscale modeling of tissue growth for cancer prognosis. $\mathrm{PhD}$ dissertation, The University of Texas at San Antonio.

Ricken, T., Schwarz, A., \& Bluhm, J. (2007). A triphasic model of transversely isotropic biological tissue with applications to stress and biologically induced growth. Computational Materials Science, 39, 124-136.

Roache, P. J. (2002). Code verification by the method of manufactured solutions. Journal of Fluids Engineering, 124, 4-10.

Roose, T., Chapman, S. J., \& Maini, P. K. (2007). Mathematical models of avascular tumor growth. SIAM Review, 49, 179-208.

Schnell, S., Grima, R., \& Maini, P. (2007). Multiscale modeling in biology new insights into cancer illustrate how mathematical tools are enhancing the understanding of life from the smallest scale to the grandest. American Scientist, 95, 134-142.

Sharp, Z. D. (2016). Personal Communication.

Tang, L., van de Ven, A. L., Guo, D., Andasari, V., Cristini, V., Li, K. C., \& Zhou, X. (2014). Computational modeling of 3d tumor growth and angiogenesis for chemotherapy evaluation. PLoS One, 9, e83962.

Truesdell, C., \& Noll, W. (2004). The non-linear field theories of mechanics. In The non-linear field theories of mechanics (pp. 1-579). Springer. 
Trusdell, C., \& Toupin, R. (1960). The classical field theories. Encyclopedia of physics, 111/1, 226-793.

Wang, M. Y., Lu, K. V., Zhu, S., Dia, E. Q., Vivanco, I., Shackleford, G. M., Cavenee, W. K., Mellinghoff, I. K., Cloughesy, T. F., Sawyers, C. L. et al. (2006). Mammalian target of rapamycin inhibition promotes response to epidermal growth factor receptor kinase inhibitors in pten-deficient and ptenintact glioblastoma cells. Cancer Research, 66, 7864-7869.

Wei, L. H., Su, H., Hildebrandt, I. J., Phelps, M. E., Czernin, J., \& Weber, W. A. (2008). Changes in tumor metabolism as readout for mammalian target of rapamycin kinase inhibition by rapamycin in glioblastoma. Clinical Cancer Research, 14, 3416-3426.

Wise, S. M., Lowengrub, J. S., Frieboes, H. B., \& Cristini, V. (2008). Threedimensional multispecies nonlinear tumor growth-i: model and numerical method. Journal of Theoretical Biology, 253, 524-543. T., Yu, J., Li, L. et al. (2007). Inactivation of yap oncoprotein by the hippo pathway is involved in cell contact inhibition and tissue growth control. Genes \& Development, 21, 2747-2761. 